University of Nebraska - Lincoln

DigitalCommons@University of Nebraska - Lincoln

\title{
Peridynamic model for dynamic fracture in unidirectional fiber- reinforced composites
}

\author{
Wenke Hu \\ University of Nebraska-Lincoln, huwenke_nbu@hotmail.com \\ Youn Doh $\mathrm{Ha}$ \\ University of Nebraska-Lincoln \\ Florin Bobaru \\ University of Nebraska-Lincoln, fbobaru2@unl.edu
}

Follow this and additional works at: https://digitalcommons.unl.edu/mechengfacpub

Part of the Mechanics of Materials Commons, Nanoscience and Nanotechnology Commons, Structural Materials Commons, and the Structures and Materials Commons

$\mathrm{Hu}$, Wenke; Ha, Youn Doh; and Bobaru, Florin, "Peridynamic model for dynamic fracture in unidirectional fiber-reinforced composites" (2012). Mechanical \& Materials Engineering Faculty Publications. 82. https://digitalcommons.unl.edu/mechengfacpub/82

This Article is brought to you for free and open access by the Mechanical \& Materials Engineering, Department of at DigitalCommons@University of Nebraska - Lincoln. It has been accepted for inclusion in Mechanical \& Materials Engineering Faculty Publications by an authorized administrator of DigitalCommons@University of Nebraska Lincoln. 


\title{
Peridynamic model for dynamic fracture in unidirectional fiber-reinforced composites
}

\author{
Wenke Hu, Youn Doh Ha, and Florin Bobaru \\ Department of Engineering Mechanics, University of Nebraska-Lincoln, Lincoln, NE 68588-0526, USA \\ Corresponding author - F. Bobaru, fbobaru2@unl.edu \\ Current Address for Y. D. Ha - Department of Naval Architecture, Kunsan National University, Republic of Korea
}

\begin{abstract}
We propose a computational method for a homogenized peridynamics description of fiber-reinforced composites and we use it to simulate dynamic brittle fracture and damage in these materials. With this model we analyze the dynamic effects induced by different types of dynamic loading on the fracture and damage behavior of unidirectional fiber-reinforced composites. In contrast to the results expected from quasi-static loading, the simulations show that dynamic conditions can lead to co-existence of and transitions between fracture modes; matrix shattering can happen before a splitting crack propagates. We observe matrix-fiber splitting fracture, matrix cracking, and crack migration in the matrix, including crack branching in the matrix similar to what is observed in recent dynamic experiments. The new model works for arbitrary fiber orientation relative to a uniform discretization grid and also works with random discretizations. The peridynamic composite model captures significant differences in the crack propagation behavior when dynamic loadings of different intensities are applied. An interesting result is branching of a splitting crack into two matrix cracks in transversely loaded samples. These cracks branch as in an isotropic material but here they migrate over the "fiber bonds" without breaking them. This behavior has been observed in recent experiments. The strong influence that elastic waves have on the matrix damage and crack propagation paths is discussed. No special criteria for splitting mode fracture (Mode II), crack curving, or crack arrest are needed, and yet we obtain all these modes of material failure as a direct result of the peridynamic simulations.
\end{abstract}

Keywords: dynamic fracture, crack propagation, fiber-reinforced composites, peridynamics, damage

\section{Introduction}

New aerospace structures, like the new Boeing-787 airplane, are incorporating more fiber-reinforced composites (FRCs) in their design [1]. The types of dynamic loading conditions that these and other similar composite structures undergo during their service life justify the need for dynamic analysis of fracture and failure in composite materials. As demonstrated by recent experimental observations [2, 3], the dynamic failure behavior of these materials is extremely complicated and a full understanding of the damage processes and mechanisms of failure in FRCs is currently lacking. Fracture in FRCs has been experimentally studied predominantly under quasi-static conditions [4-9]. Significantly fewer experiments report on dynamic fracture in FRCs. Such studies [2, 3] show that dynamic loading leads to dramatically different fracture behavior compared with quasi-static conditions.

The splitting mode (debonding between matrix and fibers) and matrix cracking are the most common intralamina fracture modes in UD FRCs under quasi-static loading [4] and they influence the other fracture modes, such as delamination and fiber breakage, as well as the ultimate strength of the composite. Dynamic experiments are conducted in Kazemahvazi et al. [2] with different strain rates from low $\left(10^{-4} \mathrm{~s}^{-1}\right)$ to high $\left(10^{2} \mathrm{~s}^{-1}\right)$ for glass/vinylester composites. The observations show that the dynamic damage behavior and failure patterns are highly sensitive to strain rates. Extensive fracture and damage produced by interconnected splitting, matrix cracking, delamination, and fiber breakage, are observed under higher strain rates. Damage and failure in S2-glass/vinylester UD composites is induced using the Split-Hopkinson-Pressure-Bar technique in [3], where the authors investigate cracking behavior under different strain rates. Haque and Ali [3] observe matrix cracking and debonding for loading UD FRCs in the transverse direction. Interestingly, at higher levels of loading the straight splitting cracks branch in the matrix in a similar way seen in isotropic materials [10] and the angle of branching (the angle between a branch and the original propagation direction) is close to $45^{\circ}$. This branching phenomenon happens in the matrix only, the fibers do not break and the matrix cracks "migrate" over the fibers. These results show that the crack path is highly sensitive to the loading conditions, and that the dynamic fracture behavior in composites is completely different from that observed under quasi-static 
loading. While limited in their number, the experimental observations for dynamic fracture in UD FRCs reveal that different fracture modes coexist when the loading is dynamic and that a complex and rich strain-rate dependent damage behavior occurs. In the present paper we propose a peridynamic model for UD FRCs and we show that this model is capable of capturing the complexity of dynamic fracture in unidirectional fiber-reinforced-composites.

Significant efforts have been made to model damage and failure in FRCs based on classical elasticity, the Finite Element Method and damage or fracture models $[8,9,11]$. For the most part, these efforts have addressed quasi-static loading conditions. Tay et al. [12] give a comprehensive review of modeling failure, delamination, and splitting in FRCs based on the element failure method. As mentioned in Guimard et al. [13], such treatments of fracture in composites require prior knowledge of the actual fracture modes and of the crack paths. As discussed above, for dynamic loading conditions the fracture modes and the crack paths are part of the solution and thus, these classical models cannot be very useful except in special situations. Recently, for example, Guimard et al. [13] used an interfacial Continuum Damage Model to study the rate effects for a setup in which a single failure mode (delamination) is active and the crack path is straight. As indicated by dynamic experiments under more general conditions [3], different failure modes coexist and are coupled in dynamic fracture of UD FRCs. The dynamic interactions among matrix cracking, splitting, delamination and stress waves determine the dynamic fracture and failure behavior in such composites. Therefore, models that intend to predict this complex behavior need to:

1. Obtain the crack paths, and their kinetics as part of the so-

lution, and

2. Allow for the autonomous interaction between stress

waves, cracks, and fracture modes.

Recently, a new nonlocal continuum model, peridynamics [14], has been proposed with the goal of solving dynamic fracture problems along the lines indicated in the two points raised above. In order to overcome mathematical inconsistencies when cracks form in the classical continuum mechanics models, peridynamics [14] uses an integral of forces (per volume-squared) over a nonlocal region around a point to replace the divergence of the stress tensor in the equations of motion. The method has been shown to correctly predict the observed phenomena in dynamic fracture in brittle materials, including crack branching, the crack propagation speed, etc. $[15,16]$ and ductile materials [17].

Other nonlocal methods have been proposed over the years [18-20], but peridynamics is unique in several important aspects: it does not contain spatial gradients and a damage model is incorporated at the micro-level, leading to a unified deformation-damage-fracture model.

Peridynamics may appear to share some similarities with the Virtual-Internal Bond (VIB) theory [21-24]: in both theories cracks can spontaneously be generated, the material "cohesion" is incorporated in the constitutive model at the bond-level. At a closer look, there are, however, significant differences: the spontaneous generation of cracks in the VIB is based on the loss of elipticity in the (classical) elastic governing equations, while in peridynamics (see Silling et al. [25]) a material instability condition, that corresponds to the ability of a discontinuous perturbation in the deformation to grow in amplitude over time, only involves the incremental material properties at each point. The VIB bonds are "local", between nearest neighbors only, and the VIB model does not introduce a length-scale [23], while peridynamics is a nonlocal formulation and the horizon introduces a material length-scale. The meaning, selection, and use of the peridynamic horizon are found in [26].
Peridynamics has been extended to model the fracture and damage of composite materials. $\mathrm{Xu}$ et al. $[27,28]$ use such a peridynamic model to simulate the damage patterns in laminated composites subjected to bi-axial loading and low-velocity impact. The details of the model are, however, not given in $\mathrm{Xu}$ et al. [27, 28]. An explicit model of fibers and matrix material is employed in Kilic et al. [29] for damage and failure in composites under quasi-static loadings. Kilic et al. [29] observed that homogenized models would not be able to capture the splitting fracture mode and thus they explicitly modeled individual fibers with peridynamics. However, the results in $[30,31]$ show that homogenized peridynamic models are able to capture localization, such as splitting fracture quite well. Explicit modeling of fibers has the advantage of obtaining the most detailed solution possible, but that comes at a high computational cost. Explicitly modeling every fiber in an actual composite structure may not be feasible due to the size of the resulting problem. In [31], we presented a homogenization-based peridynamic formulation for a unidirectional fiber-reinforced composite lamina and provided analytical formulas for the parameters used. In the discrete version of the model, scaling of the peridynamic micro-moduli is required in order to maintain the strain energy of the numerical model under grid refinement the same. For a center-cut UD composite lamina with the computational grid aligned with the fibers under dynamic tensile longitudinal loading, convergence is shown for the splitting mode fracture in terms of decreasing the nodal spacing for a fixed nonlocal region. Also, as the nonlocal region decreases, the peridynamic results for the splitting crack propagation speed tend to the analytical results for a dynamic interface debonding problem. The results also pointed to the strong influence stress waves have on the splitting crack propagation speed.

In the present contribution we extend the discrete model proposed in [31] so that it is valid for modeling dynamic fracture in a general case, in which the discretization grid has an arbitrary orientation relative to the fiber orientation in the UD FRC. The discrete model in [31] does not preserve the strain energy of the material if the uniform discretization grid is not aligned with the fibers. While using uniform grids aligned with the fiber direction is a natural choice when discretizing FRCs, in certain situations, like adaptive grid refinement [32], unstructured grids are needed. We introduce an algorithm that allows for random or unstructured discretizations to be used. We employ this model to compute, in particular, the dynamic fracture and damage behavior when the grid orientation relative to the fibers is $45^{\circ}, 90^{\circ}$, and some arbitrary value $\left(25^{\circ}\right)$ in a center-cut lamina under shock-like loading conditions applied along the $0^{\circ}$ direction. We provide the semi-analytical derivation for the scaling factors required for the discrete formulation in the special case when the angle between the fiber orientation and the uniform grid is $45^{\circ}$, while for the general case of fiber orientation and for non-uniform/random discretizations, a new algorithm is introduced. The scaling is based on the requirement that the strain energy density of the discrete peridynamic model matches the classical strain energy density of the composite under homogeneous longitudinal and transverse deformation. We show that the peridynamic simulations capture the autonomous interaction between stress waves and the crack propagation behavior in UD FRC laminas loaded dynamically, and that the failure and damage patterns and fracture modes follow remarkably well some recent experimental observations [3] on the strain-rate dependence of cracks in dynamically loaded UD composites.

The paper is organized as follows: In Section 2, we briefly review the basic formulation for peridynamics and the homogenized peridynamic composite lamina model. In Section 3 we derive scaling factors the new discrete model for the spe- 
cial case when the fiber direction makes an angle of $45^{\circ}$ with the orientation of a uniform grid, and present a new algorithm for computing the scaling factors in the general case of an arbitrary orientation between the fiber direction and a structured or unstructured grid. In Section 4, we treat a number of examples of dynamic fracture in UD FRCs and analyze, in particular, the influence of the stress waves (generated by the suddenly applied loads or reflected from the boundaries) on the crack propagation and damage patterns. We use two different loading cases, vary the loading amplitude, and compare the peridynamic results with experiments. We perform a convergence study for arbitrary fiber orientation by using the proposed algorithm. Conclusions are given in Section 5.

\section{The homogenized peridynamic composite model}

\subsection{Review of the peridynamic formulation}

The peridynamic theory uses an integral of forces (per unit volume squared) acting at a point over a certain "horizon" (a nonlocal region around the point) instead of the divergence of stresses term in the classical equations of motion [14]. Since spatial differentiation is eliminated from the mathematical framework of peridynamics, this formulation is well suited for modeling problems in which displacement discontinuities emerge, interact, and evolve in time. The peridynamic equations of motion are:

$$
\rho \ddot{\mathbf{u}}(\mathbf{x}, t)=\int_{\mathcal{H}} \mathbf{f}(\mathbf{u}(\hat{\mathbf{x}}, t)-u(\mathbf{x}, t), \hat{\mathbf{x}}-\mathbf{x}) d V_{\hat{\mathbf{x}}}+\mathbf{b}(\mathbf{x}, t),
$$

where $\mathbf{f}$ is the pairwise force function in the peridynamic bond that connects point $\hat{\mathbf{x}}$ to $\mathbf{x}$, and $\mathbf{u}$ is the displacement vector field. $\rho$ is the mass density and $\mathbf{b}(\mathbf{x}, t)$ is the body force acting at $\mathbf{x}$ at time $t$. The integral is defined over a region $\mathcal{H}$ called the "horizon." The region is taken here to be a circle of radius $\delta$, but its shape can be arbitrary. Further comments about the horizon can be found in [26].

A micro-elastic material is defined as one for which the pairwise force derives from a micropotential $\omega$ [14]:

$$
\mathbf{f}(\boldsymbol{\eta}, \xi)=\frac{\partial \omega(\boldsymbol{\eta}, \xi)}{\partial \boldsymbol{\eta}}
$$

where $\boldsymbol{\xi}$ and $\mathbf{\eta}$ are the relative position and relative displacement of two points $\hat{\mathbf{x}}$ and $\mathbf{x}$ in the reference configuration.

The strain energy density at a given point is

$$
W(\mathbf{x})=\frac{1}{2} \int_{\mathcal{H}} \omega(\boldsymbol{\eta}, \xi) d V_{\xi}
$$

The 1/2 factor in Equation (3) is present because the elastic energy in a bond is shared by the two nodes connected by the bond. A "linear" micro-elastic potential, which leads to a linear relationship between the bond force and the relative elongation of the bond, is obtained if we take

$$
\omega(\boldsymbol{\eta}, \xi)=\frac{c(\xi) s^{2} \xi}{2}
$$

where $\xi=\|\xi\|$ and $s$ is the bond relative elongation:

$$
s=\frac{\|\xi+\boldsymbol{\eta}\|-\xi}{\xi}
$$

The corresponding pairwise force becomes

$$
\mathbf{f}(\boldsymbol{\eta}, \xi)=\frac{\partial \omega(\boldsymbol{\eta}, \xi)}{\partial \boldsymbol{\eta}}=c(\xi) s\|\xi\| \frac{\partial s}{\partial \boldsymbol{\eta}}=c(\xi) s \frac{\partial\|\boldsymbol{\xi}+\boldsymbol{\eta}\|}{\partial \boldsymbol{\eta}}
$$

with

$$
\frac{\partial\|\boldsymbol{\xi}+\boldsymbol{\eta}\|}{\partial \boldsymbol{\eta}}=\frac{\boldsymbol{\xi}+\boldsymbol{\eta}}{\|\boldsymbol{\xi}+\boldsymbol{\eta}\|}=e
$$

$e$ is the unit vector along the direction of vector $\xi+\eta$, the bond between $\hat{\mathbf{x}}$ and $\mathbf{x}$ in the deformed configuration.

The function $c(\xi)$ is called the micromodulus function and represents the bond elastic stiffness. This function is required to satisfy certain conditions of regularity; however, the set of allowable functions is quite large [33]. For isotropic materials, the micromodulus function versions is computed by matching the peridynamic strain energy density to the classical strain energy density (see [34] for 1D, [15] for 2D, and [35] for 3D).

\subsection{A homogenized peridynamic model for unidirectional FRCs}

In [31] we introduced a new homogenized peridynamic model for fiber-reinforced UD composites. The parameters in the model are obtained based on matching strain energy densities, for a homogeneous deformation, between the classical formulation and the peridynamic formulation. The total strain energy density for the homogenized anisotropic peridynamic material at a point has contributions from the fibers (along the longitudinal direction) and the matrix (from all other directions) as schematically indicated in Figure 1. The peridynamic bonds at a point in the homogenized anisotropic peridynamic material have different bond stiffness along the longitudinal direction than along all other directions. In peridynamics, every material point is connected to points within its horizon through peridynamic bonds.

We call the peridynamic bonds aligned with the fiber direction "fiber bonds" and all other bonds "matrix bonds." It is important to emphasize that the "fiber bonds" properties are not matched to the actual fiber properties, but are calibrated to the analytical effective elastic properties of the composite lamina along the fiber direction. The same is true for the "matrix bonds."

To compute the strain energy density along the longitudinal deformation we need to make use of the Dirac-delta function. For a given homogeneous deformation, we match the strain energy of the material along the longitudinal direction with the strain energy of homogenized anisotropic peridynamic material along the same direction. Along the transverse direction we do the same.

Given a homogeneous bi-axial deformation of size $s$ aligned with the longitudinal and the transverse directions, the peridynamic elastic strain energy density of the UD composite can be written as:

$$
\begin{aligned}
& W_{1}=\frac{1}{2} \int_{\mathcal{H}} \omega_{\mathrm{fb}}(\boldsymbol{\eta}, \xi)[D(\xi, 0)+D(\xi, \pi)] d A_{\xi}=\frac{1}{2} \int_{-\delta}^{\delta} \omega_{\mathrm{fb}}(\boldsymbol{\eta}, \xi) d \xi \\
& W_{2}=\frac{1}{2} \int_{\mathcal{H}} \omega_{\mathrm{mb}}(\boldsymbol{\eta}, \xi) d A_{\xi}
\end{aligned}
$$

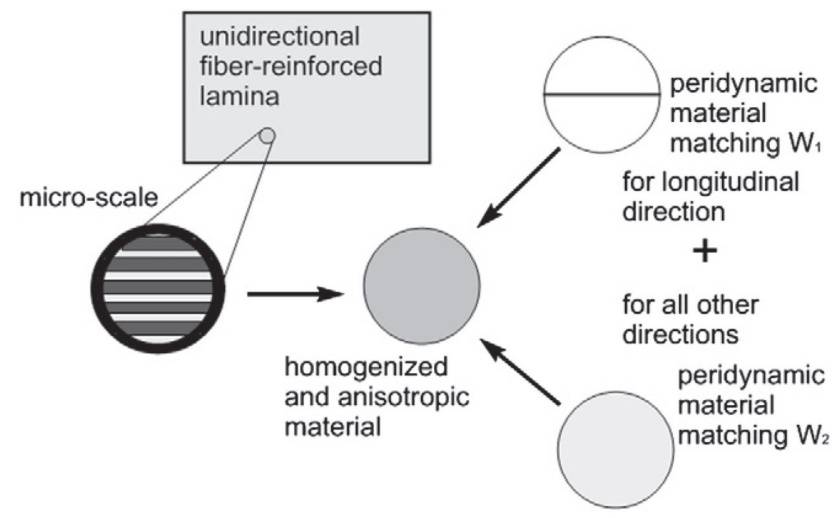

Figure 1. Schematic of the homogenization procedure [31]. 


$$
\omega_{\mathrm{fb}}(\boldsymbol{\eta}, \xi)=\frac{\bar{c}_{\mathrm{fb}}(\xi) s^{2} \xi}{2}, \quad \omega_{\mathrm{mb}}(\boldsymbol{\eta}, \xi)=\frac{\bar{c}_{\mathrm{mb}}(\xi) s^{2} \xi}{2}
$$

where $s$ is the constant strain value of the homogeneous deformation (see also Equation (5)). $\omega_{\mathrm{fb}}$ and $\omega_{\mathrm{mb}}$ are the micro-elastic potentials for the anisotropic peridynamic material along the longitudinal and transverse directions. $\bar{c}_{\mathrm{fb}}(\xi)$ and $\bar{c}_{\mathrm{mb}}(\xi)$ are the elastic stiffness (micromodulus) functions for "fiber bonds" and "matrix bonds" respectively. $D(\xi, 0)$ and $D(\xi, \pi)$ are the two-dimensional Dirac delta functions (distributions) for the polar coordinates $\theta=0$ and $\theta=\pi\left(\theta=\arctan \left(\xi_{2} \div \xi_{1}\right)\right.$, $\left.\xi=\left(\xi_{2}, \xi_{1}\right)=(\xi, \theta)\right)$.

The classical strain energy density for the homogenized composite lamina under 2D plane stress conditions with the same homogenous deformation is given by:

$W_{1}^{\text {classical }}=\frac{1}{2} \frac{\left(E_{11}+v_{12} E_{22}\right)}{\left(1-v_{12} v_{21}\right)} s^{2}, \quad W_{2}^{\text {classical }}=\frac{1}{2} \frac{\left(E_{22}+v_{12} E_{22}\right)}{\left(1-v_{12} v_{21}\right)} s^{2}$

We obtain the micromodulus functions for "fiber bonds" and "matrix bonds" by matching the peridynamic strain energy density to the classical strain energy density in a homogenized UD FRC as follows:

$$
\begin{aligned}
& \bar{c}_{\mathrm{fb}}(\xi)=\frac{6\left(E_{11}+v_{12} E_{22}\right)}{\left(1-v_{12} v_{21}\right) \delta^{2}}\left(1-\frac{\xi}{\delta}\right) \\
& \bar{c}_{\mathrm{mb}}(\xi)=\frac{12\left(E_{22}+v_{12} E_{22}\right)}{\left(1-v_{12} v_{21}\right) \pi \delta^{3}}\left(1-\frac{\xi}{\delta}\right)
\end{aligned}
$$

where $E_{11}$ and $E_{22}$ are the longitudinal and transverse elastic Young's modulus in the principal material axes, respectively. $v_{12}$ is the longitudinal Poisson's ratio and $v_{21}$ is the transverse Poisson's ratio. Notice that in the bond-based version we are only able to effectively match the $E_{11}$ and $E_{22}$ parameters for the unidirectional composite. All other material parameters (Poisson ratios, shear moduli) are preset by these two values. Therefore, the bond-based peridynamic model will match some, but not all composites. A state-based peridynamic formulation would eliminate these restrictions (see [36]). Nevertheless, from the point of view of the ability of modeling dynamic fracture and damage in a unidirectional FRC, how well the shear moduli and Poisson ratios are represented have a secondary importance.

Assuming that the fibers are along the $x$-direction (see Figure 2), the micromodulus for the homogenized anisotropic peridynamic material, for modeling unidirectional FRCs, in $(\xi, \theta)$, is:

$$
C(\xi, \theta)= \begin{cases}\bar{c}_{\mathrm{fb}}(\xi) & \text { if } \theta=0 \text { or } \pi \\ \bar{c}_{\mathrm{mb}}(\xi) & \text { otherwise }\end{cases}
$$

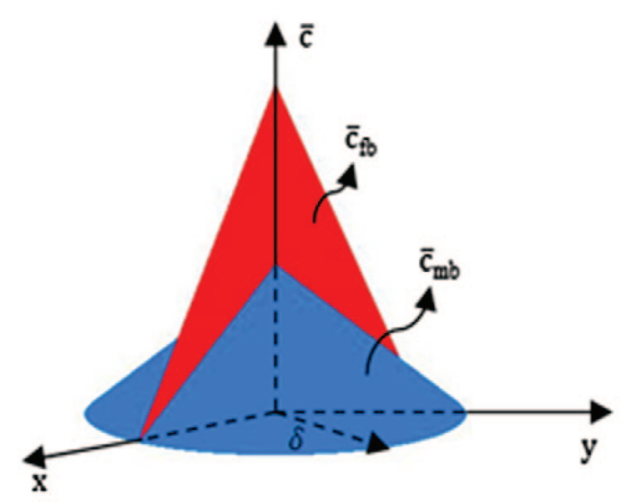

Figure 2. The conical micromodulus function for the homogenized peridynamic model of a unidirectional fiber-reinforced composite.
The pairwise force function $\mathbf{f}$ for our model can be written as follows:

$$
\mathbf{f}(\boldsymbol{\eta}, \boldsymbol{\xi})= \begin{cases}\mathbf{f}_{\mathrm{fb}}(\boldsymbol{\eta}, \xi)[D(\xi, 0)+D(\xi, \pi)] & \text { if } \theta=0 \text { or } \pi \\ \mathbf{f}_{\mathrm{mb}}(\boldsymbol{\eta}, \xi) & \text { otherwise }\end{cases}
$$

where $\mathbf{f}_{\mathrm{fb}}(\boldsymbol{\eta}, \boldsymbol{\xi})$ and $\mathbf{f}_{\mathrm{mb}}(\boldsymbol{\eta}, \boldsymbol{\xi})$ are the pairwise force functions for "fiber bonds" and "matrix bonds" respectively.

The history-dependent damage model in peridynamics consists in breaking the peridynamic bonds when the relative elongation (see Equation (5)) between the nodes exceeds a critical value $s_{0}[14,35]$. The critical relative elongation $s_{0}$ at the micro-scale is obtained from the macro-scale measurable fracture energy $\left(G_{0}\right)$ (see [15] for the 2D case and [35] for the 3D case, for isotropic materials).

For the homogenized peridynamic model of UD FRCs, we postulate that damage occurs in the "fiber bonds" and "matrix bonds" when they are stretched beyond some corresponding critical relative elongations, $s_{0}^{\mathrm{fb}}$ and $s_{0}^{\mathrm{mb}}$ [31]. The critical relative elongation $s_{0}^{\mathrm{fb}}$ is obtained by matching the work needed to break all bonds in a peridynamic material made entirely out of "fiber bonds," across a line, for the 2D case (and a surface in the $3 \mathrm{D}$ case), to the fracture energy $G_{0}^{11}$ (Mode I intralamina fracture energy for longitudinal loading). The value for $s_{0}^{\mathrm{mb}}$ is obtained in a similar way by matching $G_{0}^{22}$ (Mode I intralamina fracture energy for transverse loading) with the work needed to break all bonds in a material made entirely out of "matrix bonds," across a line in 2D (and a surface in 3D). The values we obtain are:

$$
s_{0}^{\mathrm{fb}}=\sqrt{\frac{10 \pi G_{0}^{11}}{\bar{c}_{\mathrm{fb}} \delta^{3}}}, \quad s_{0}^{\mathrm{mb}}=\sqrt{\frac{20 G_{0}^{22}}{\bar{c}_{\mathrm{mb}} \delta^{4}}}
$$

For simplicity, in the present work, the critical relative elongation $s_{0}$ and $s_{0}$ mb only depend on the material properties of UD FCRs, such as $E_{11}, E_{22}, G_{0}^{11}, G_{0}^{22}$, etc., and on the horizon size. Note that other dependencies may be introduced, for example on the current damage state [16], on various environmental and/or manufacturing parameters, and on time [35].

\subsection{Convergence in peridynamics}

In peridynamics one can introduce two types of numerical convergence, see Figure 3: $\delta$-convergence, in which one takes the horizon size to zero while keeping the number of nodes inside the horizon region the same (thus increasing the overall grid density), and the $m$-convergence where one maintains the horizon size fixed while increasing the grid density. In $\delta$-convergence we expect the nonlocal solutions to converge to the classical, local solutions (see [34,37]). On the other hand, under $m$-convergence we approach the exact nonlocal solution for the particular horizon size selected. The $m$-convergence and $\delta$-convergence results in [31] for the special case when the grid is aligned with the fibers, led to the conclusion that a value of $m=5(m=\delta / \Delta x$, where $\Delta x$ is the grid spacing) provides a good balance between accuracy and computational cost and a horizon of about $2 \mathrm{~mm}$ is able to capture the damage types and the main features of the damage evolution processes, for the sample sizes tested. The crack propagation speed for a splitting crack induced via shock loading from the boundaries (see [31]) is influenced by the wave dispersion and reflection from the boundaries and other crack surfaces. The horizon size determines the wave dispersion in peridynamics and computational tests [31] indicate that a horizon of less than $1 \mathrm{~mm}$ needs to be used in order to match some analytical results for a dynamic debonding crack. In the present study we analyze qualitatively the evolution of damage and are interested in observing the effects the horizon size has on the evolution of damage and crack propagation. For the general case, when the grid and fibers are not is any special orientation, we investigate both $m$ - and $\delta$-convergence. 


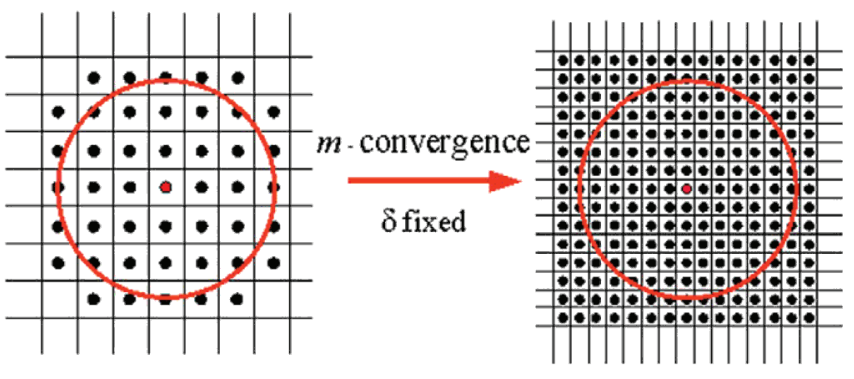

(a) $m$-convergence

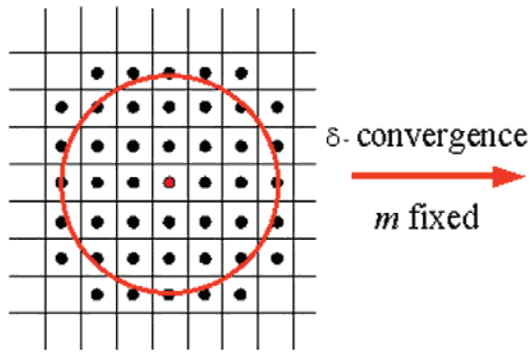

(b) $\delta$-convergence

Figure 3. Schematic description of $m$-convergence and $\delta$-convergence. $m=\delta / \Delta x$, where $\Delta x$ is the grid spacing. (a) $m$-convergence, (b) $\delta$-convergence.

\section{The scaled discrete peridynamic model for arbitrary fiber orientation relative to the discretization grid}

In [31] we used the model described above with a uniform discretization grid aligned with the fiber directions and performed calculations on a $0^{\circ}$ lamina with a center crack perpendicular to the fiber direction. A certain scaling of the micromodulus function was necessary in order to keep the strain energy density from the discretized homogenized peridynamic model the same under grid refinement, as is the case when one performs $m$-convergence studies (see Table 2 in [31]). The discrete version of the conical micromodulus function for the continuum anisotropic (UD FRC) peridynamic model is [31]:

$$
C^{d}(\xi, \theta)= \begin{cases}\bar{\lambda}_{\mathrm{fb}} \bar{c}_{\mathrm{fb}}(\xi) & \text { if } \theta=0 \text { or } \pi \\ \bar{\lambda}_{\mathrm{mb}} \bar{c}_{\mathrm{mb}}(\xi) & \text { otherwise }\end{cases}
$$

where $\bar{\lambda}_{\mathrm{fb}}$ and $\bar{\lambda}_{\mathrm{mb}}$ are the corresponding scaling factors for fiber bonds and matrix bonds, for the case when a uniform grid aligned with the fiber direction is employed. These factors are related to the discretization size. The dimension for $\bar{\lambda}_{\mathrm{fb}}$ is $\mathrm{m}^{-1}$ while $\bar{\lambda}_{\mathrm{mb}}$ is dimensionless. Alternatively, in order to have both scaling factors dimensionless, we can re-write the homogenized micromodulus for the UD FRC based on the conical micromodulus function of two isotropic peridynamic materials made out of "fiber bonds" and "matrix bonds" (see [31] for details) as follows:

$$
\begin{aligned}
C^{d}(\xi, \theta) & = \begin{cases}\lambda_{\mathrm{fb}} C_{11}^{\text {iso }}(\xi) & \text { if } \theta=0 \text { or } \pi \\
\lambda_{\mathrm{mb}} C_{22}^{\text {iso }}(\xi) & \text { otherwise }\end{cases} \\
c_{11}^{\text {iso }}(\xi) & =\frac{12\left(E_{11}+v_{12} E_{22}\right)}{\left(1-v_{12} v_{21}\right) \pi \delta^{3}}\left(1-\frac{\xi}{\delta}\right) \\
c_{22}^{\text {iso }}(\xi) & =\frac{12\left(E_{22}+v_{12} E_{22}\right)}{\left(1-v_{12} v_{21}\right) \pi \delta^{3}}\left(1-\frac{\xi}{\delta}\right)
\end{aligned}
$$

with $\lambda_{\mathrm{fb}}$ and $\lambda_{\mathrm{mb}}$ being scaling factors corresponding to the "fiber bonds" and the "matrix bonds". The connections between $\lambda_{\mathrm{fb}}$ and $\bar{\lambda}_{\mathrm{fb}}$, and $\lambda_{\mathrm{mb}}$ and $\bar{\lambda}_{\mathrm{mb}}$ are given in [31]. We call this model (developed in [31]) the Homogenized-Discrete-Peridynamic-zero-degree-fiber model, or, in short, the HD-Pod model.

The scaling factors obtained for the HD-Pod model do not apply for the case when the uniform discretization grid is not aligned with the fiber orientation or for the case of non-uniform discretizations. Indeed, the "fiber bonds" area (the total area of nodes that have "fiber bonds" connections with the current node) is different for different angles between the fiber direction and the orientation of the discretization grid. For instance, as shown in Figure 4, for a given uniform discretization, the "fiber bonds" area for $45^{\circ}$ fiber orientation (green areas) is different from the "fiber bonds" area for $0^{\circ}$ fiber orientation (red areas). $\varphi$ is the angle between the grid and the fiber orientation. Notice that the "fiber bonds" areas include the

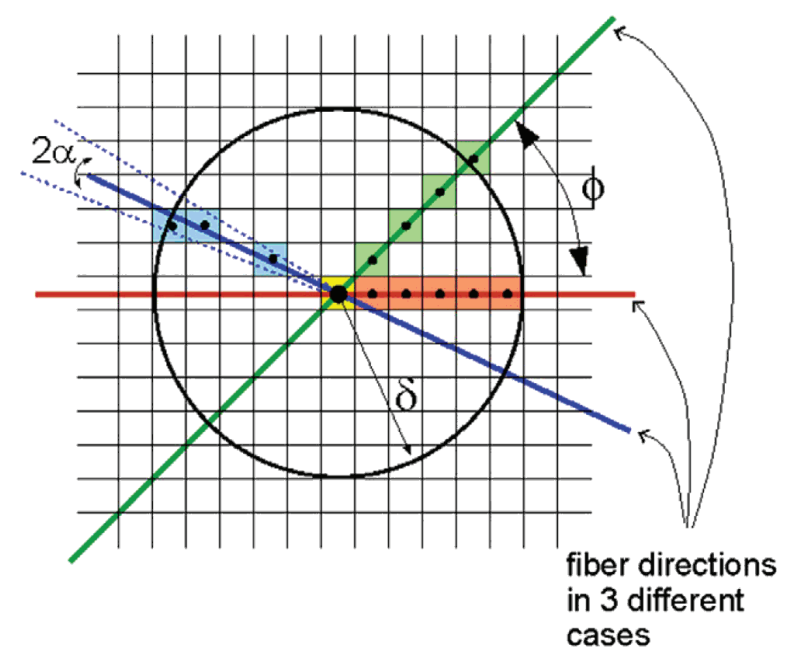

Figure 4. The discrete peridynamic model for three different unidirectional FRCs at a node for a uniform grid. Possible orientations between the grid and the fibers are shown (red, green and blue represent $\varphi=0^{\circ}, 45^{\circ}$, and some arbitrary value). The nodes with fiber bond connections to the central node are marked in each case.

area of the center node 1 . As a result of the "fiber bonds" area changing when the fiber orientation relative to some given discretization grid changes, the strain energy density at a node will change. For example, we compute the strain energy density at the central node in a $0.1 \mathrm{~m} \times 0.2 \mathrm{~m}$ sample with a discretization of $\Delta x=0.083 \mathrm{~mm}$ and $\delta=1 \mathrm{~mm}$ and an imposed homogeneous longitudinal and transverse deformation of same magnitudes $(s=0.005)$ for $\varphi=45^{\circ}$ and $\varphi=0^{\circ}$. The strain energy density should be the same between these two cases. The reason for the particular horizon size and discretization size used here can be found in the convergence study performed in [31] for $\varphi=0^{\circ}$. Table 1 shows the changes in the strain energy density, for a uniform discretization, induced by the different fiber orientation relative to the grid orientation. A proper scaling is needed therefore to keep the strain energy density independent of the fiber orientation relative to the grid orientation. We will also provide an algorithm that deals with this issue for the case of random or unstructured discretizations.

In what follows, we use a semi-analytical approach to estimate the scaling required for the case in which the fibers make a $45^{\circ}$ angle with the uniform discretization grid (since this is the other special orientation with the uniform grid besides the 0 or 90 cases, see Figure 4). For the general grid orientation relative to a uniform grid and/or a non-uniform discretization such an approach is not possible and, instead, we introduce a new algorithm that correctly scales the model. We will refer to this more general discrete model as the Homogenized-Discrete-Peridynamic-arbitrary-degree-fiber-orientation model (in short the HD-Pad model). 
Table 1. Comparison of strain energy densities for $0^{\circ}$ and $45^{\circ}$ grid orientation relative to the fibers and a homogeneous deformation (equal relative elongation $s=0.005$ along longitudinal and transverse directions) obtained with the HD-Pod and HD-Pad models with $\delta=1 \mathrm{~mm}$ and $m=12$.

\begin{tabular}{|c|c|c|c|c|}
\hline & \multicolumn{3}{|c|}{ Values computed with the peridynamic model } & \multirow{2}{*}{$\begin{array}{l}\text { Analytical values from the } \\
\text { classical (local) model }\end{array}$} \\
\hline & $\begin{array}{l}\varphi=0 \\
(\text { HD-Pod model) }\end{array}$ & $\begin{array}{l}\varphi=45^{\circ} \\
\text { (HD-Pod model) }\end{array}$ & $\begin{array}{l}\varphi=45^{\circ} \\
\text { (HD-Pad model) }\end{array}$ & \\
\hline "Fiber-bonds" contribution to strain energy density $\left(\mathrm{MJ} / \mathrm{m}^{2}\right)$ & 4.1133 & 2.9112 & 4.1166 & 4.1459 \\
\hline "Matrix-bonds" Contribution to strain energy density $\left(\mathrm{MJ} / \mathrm{m}^{2}\right)$ & 0.1000 & 0.1016 & 0.1010 & 0.1002 \\
\hline Total strain energy density $\left(\mathrm{MJ} / \mathrm{m}^{2}\right)$ & 4.2133 & 3.0128 & 4.2176 & 4.2461 \\
\hline
\end{tabular}

\subsection{Semi-analytical derivation of the new discrete model and the scaling factors for $\varphi=45^{\circ}$ and uniform discretization}

In practical application of laminated composites, the most widely selected fiber orientations are $0^{\circ}, 90^{\circ}$, and $45^{\circ}$ plies. The HD-Pod model proposed in [31] can be used for the angle between the grid and the fiber direction of $0^{\circ}$ (or $90^{\circ}$ ). In order to have a discrete model that is independent not only on the discretization size but also on the orientation of the discrete grid relative to the fibers we need additional scaling factors, as discussed above and seen from Table 1 . In this section, we provide a semi-analytical derivation for the scaling factors in the discrete peridynamic model for the case when the angle between the uniform grid and the fibers is $45^{\circ}$. In this case (uniform grid and $\varphi=45^{\circ}$ ), the geometry allows to easily derive the scaling parameters (see nodes with green areas in Figure 4). The nodes on the diagonal direction $\left(45^{\circ}\right)$ of the grid cells have "fiber-bonds" connections with the center node. The goal is to obtain the same strain energy density (under a homogeneous and equal deformation imposed along the longitudinal and transverse directions) for $\varphi=45^{\circ}$ as we do with the HD-Pod model for the $\varphi=0^{\circ}$ case (which is the same with the classical value of the strain energy density). For all other orientations, like the $25^{\circ}$ orientation shown in Figure 4, we propose a numerical algorithm that computes the corresponding scaling parameters in the model (see Section 3.2). This general case will also be applicable to random or non-uniform grids.

Let $W_{\mathrm{fb} 00}^{\mathrm{d}}$ and $W_{\mathrm{fb} 45}^{\mathrm{d}}$ be the discrete elastic strain energy densities from the "fiber bonds" for $\varphi=0^{\circ}$ (red line in Figure 4) and $\varphi=45^{\circ}$ (green line in Figure 4) for the given longitudinal and transverse deformations with a constant relative elongation $s$ (see Equation (5)), respectively:

$$
\begin{aligned}
& W_{\mathrm{fb} \_0}^{\mathrm{d}}=\frac{1}{2} \int_{A_{\mathrm{fb} \_}^{\mathrm{d}} 0} \frac{c_{11}^{\mathrm{iso}}(\xi) s^{2} \xi}{2} d A_{\xi} \\
& W_{\mathrm{fb} \_45}^{\mathrm{d}}=\frac{1}{2} \int_{A_{\mathrm{fb} \_45}^{\mathrm{d}}} \frac{c_{11}^{\mathrm{iso}}(\xi) s^{2} \xi}{2} d A_{\xi}
\end{aligned}
$$

where $A_{\mathrm{fb} \_0}^{\mathrm{d}}$ and $A_{\mathrm{fb} \_45}^{\mathrm{d}}$ are the "fiber bonds" areas (red and green areas in Figure 4) for $\varphi=0^{\circ}$ and $\varphi=45^{\circ}$, respectively. Obviously, $W_{\mathrm{fb} \_0}^{\mathrm{d}}$ and $W_{\mathrm{fb} \_45}^{\mathrm{d}}$ will not match each other since the areas of integration are different (see also Table 1). We introduce the scaling factor $\gamma_{\mathrm{fb}}$ for the "fiber bonds" defined by

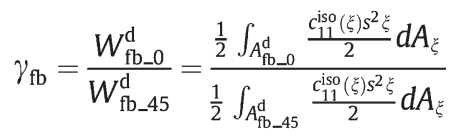

Using mid-point numerical integration for the conical micromodulus function (see Equation (18)), the scaling factor $\gamma_{\mathrm{fb}^{\prime}}$, for a given discretization, becomes:

$$
\gamma_{\mathrm{fb}}=\frac{W_{\mathrm{fb} \_0}^{\mathrm{d}}}{W_{\mathrm{fb} \_45}^{\mathrm{d}}} \cong \frac{\sum_{i=1}^{p}\left(\xi_{i}-\frac{\xi_{i}^{2}}{\delta}\right) A_{i}}{\sum_{j=1}^{q}\left(\xi_{j}^{\prime}-\frac{\xi_{j}^{\prime 2}}{\delta}\right) A_{j}}
$$

where $A_{i}$ is the nodal area; here, for simplicity, we assume that all the nodes inside the horizon have their full volume contained in the horizon; also, the distances from the center node to the $i$ th and $j$ th nodes on the $0^{\circ}$ and $45^{\circ}$ directions are:

$$
\xi_{i}=i \Delta x, \quad \xi_{j}^{\prime}=j \sqrt{2} \Delta x
$$

where $\Delta x$ is the grid spacing and $p$ and $q$ are the number of nodes along $\varphi=0^{\circ}$ and $\varphi=45^{\circ}$ directions inside the horizon $\delta$ :

$$
p=\left[\frac{\delta}{\Delta x}\right], \quad q=\left[\frac{\delta}{\sqrt{2} \Delta x}\right]
$$

The notation $\left[\frac{\delta}{\Delta x}\right]$ defines the integer part of $\frac{\delta}{\Delta x}$. From Equations (22), (23), and (24), we obtain:

$$
\gamma_{\mathrm{fb}} \cong \frac{\frac{1}{p^{2}} \sum_{i=1}^{p}\left(p * i-i^{2}\right)}{\frac{1}{q^{2}} \sum_{j=1}^{q}\left(q * j-j^{2}\right)}=\frac{p+2-\frac{2}{p}-\frac{1}{p^{2}}}{q+2-\frac{2}{q}-\frac{1}{q^{2}}}
$$

If $p$ and $q$ are sufficiently large and the horizon $\delta$ is constant, the scaling factor $\gamma_{\mathrm{fb}}$ is well approximated by:

$$
\gamma_{\mathrm{fb}} \cong \frac{p}{q} \cong \sqrt{2}=\frac{1}{\cos 45^{\circ}}
$$

Similarly, let $W_{\text {mb_0 }}^{\mathrm{d}}$ and $W_{\text {mb_45 }}^{\mathrm{d}}$ be the discrete elastic strain energy densities from the "matrix bonds" for $\varphi=0^{\circ}$ and $\varphi=45^{\circ}$, for the given longitudinal and transverse deformations with a constant relative elongation $s$ (see Equation (5)), respectively. We find the scaling factor $\gamma_{\mathrm{mb}}$, for "matrix bonds" as:

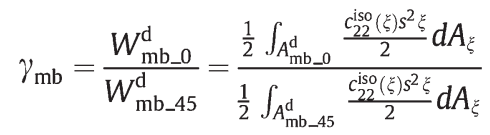

where $A_{\text {mb } 00}^{\mathrm{d}}$ and $A_{\mathrm{mb} \_45}^{\mathrm{d}}$ are the "matrix bonds" areas (the entire horizon area minus the "fiber bonds" area) for $\varphi=0^{\circ}$ and $\varphi=45^{\circ}$, respectively.

Following the same procedure as before, the scaling factor $\gamma_{\mathrm{mb}}$ for "matrix bonds" is

$$
\gamma_{\mathrm{mb}} \cong\left(\frac{\pi \delta-\Delta x}{\pi \delta-\Delta x / \gamma_{\mathrm{fb}}}\right)
$$

The new discrete version of the conical micromodulus functions for $\varphi=45^{\circ}$ is therefore (see also Equation (17)):

$$
C_{45}^{\mathrm{d}}(\xi, \theta)= \begin{cases}\gamma_{\mathrm{fb}} * \lambda_{\mathrm{fb}} c_{11}^{\text {iso }}(\xi) & \text { if } \theta= \pm 45 \\ \gamma_{\mathrm{mb}} * \lambda_{\mathrm{mb}} C_{22}^{\text {iso }}(\xi) & \text { otherwise }\end{cases}
$$

We use this scaled micromodulus to compute the peridynamic solution for the test problem described just above Section 3.1. As shown in Table 1, the strain energy density based on HDPad model for $\varphi=45^{\circ}$ now matches the classical result well. Note that we are using approximate spatial integration of the peridynamic equations, thus the strain energy density with the discrete peridynamic model will not exactly match the classical strain energy density. 


\subsection{An algorithm for computing scaling factors in the discrete peridynamic model for arbitrary grids}

For an arbitrary orientation of the grid relative to the fibers and/or when a non-uniform discretization is used, there might not be any nodes exactly sitting along the fiber direction that passes through the center node (see Figure 4). To define "fiber bonds" in these cases we search in a "cone" of angle $2 a$ about the fiber direction, where $a$ is a given tolerance. If angle $\varphi^{\prime}$ between a peridynamic bond direction belonging to the center node and the horizontal direction falls in the interval $[\varphi-\alpha, \varphi+\alpha]$, then that bond is considered a "fiber bond" (see, for example, the blue nodes in Figure 4). All other bonds are considered to be "matrix bonds." Explicit formulations for scaling factors $\gamma_{\mathrm{fb}}$ and $\gamma_{\mathrm{mb}}$ in these cases are not easy to obtain because of the dependence on: the fiber orientation relative to the grid, the selected angle tolerance, the grid spacing and the horizon size, and more importantly, on the specific discretization used in the case of a non-uniform or randomnodes discretization. We obtain therefore appropriate scaling factors for "fiber bonds" and "matrix bonds" numerically. We compute the strain energy density for "fiber bonds" and "matrix bonds" for a specific fiber orientation $\varphi$ and under given homogeneous longitudinal and transverse deformations, and match them to the longitudinal and transverse components of the classical strain energy density $\left(W_{1}\right.$ and $\left.W_{2}\right)$ by the following procedure, given in Algorithm 1.

Algorithm 1. Numerical evaluation of the scaling factors for arbitrary uniform grid orientation relative to the fibers and/or an arbitrary discretization.

1: Input fiber orientation $\varphi$ (relative to the horizontal axis) and angle tolerance $\alpha$

2: Impose deformation along longitudinal and transverse direction

3: Compute longitudinal and transverse classical strain energy densities $W_{1}$ and $W_{2}$

4: Compute the angle $\varphi^{\prime}$ between a peridynamic bond direction and horizontal direction

5: If $\varphi-\alpha \leq \varphi^{\prime} \leq \varphi+\alpha$ then

6: the bond is assigned as "fiber bond"

7: Else

8: the bond is assigned as "matrix bond"

9: $\quad$ Endif

10: Compute the peridynamic discrete strain energy densities for "fiber bonds" $W_{\mathrm{fb} \_\varphi}^{\mathrm{d}}$ and "matrix bonds" $W_{\mathrm{mb} \_\varphi}^{\mathrm{d}}$

11: Compute the scaling factors for "fiber bonds" and

$$
\text { "matrix bonds": } r_{\mathrm{fb}}^{\varphi}=\frac{W_{1}}{W_{\mathrm{fb} \_\varphi}^{\mathrm{d}}} \quad \text { and } \quad r_{\mathrm{mb}}^{\varphi}=\frac{W_{2}}{W_{\mathrm{mb} \_\varphi}^{\mathrm{d}}}
$$

The tolerance angle $\alpha$ may be chosen in terms of $m$, the ratio between the horizon and the grid spacing. For example, if $m$ is large, the angle tolerance $\alpha$ can be small. In fact, in the limit of $m$ going to infinity, the value of $\alpha$ could be taken as the actual fiber misalignment in the manufactured UD FRCs. For instance, in a carbon fiber composite material like the APC-2 [38], most of the fibers are found to lie within $\pm 3^{\circ}$ of the fiber direction.

The scaling factors obtained from Algorithm 1 are then used to scale the micro-modulus (conical) function as follows:

$$
C_{\phi}^{\mathrm{d}}(\xi, \theta)= \begin{cases}\gamma_{\mathrm{fb}}^{\phi} c_{11}^{\text {iso }}(\xi) & \text { if } \theta=\varphi, \\ \gamma_{\mathrm{mb}}^{\phi} \mathrm{c}_{22}^{\text {iso }}(\xi) & \text { otherwise }\end{cases}
$$

This micromodulus function is then used in the peridynamic computations that use a uniform grid with an arbitrary orientation to the fibers, or that use an arbitrary discretization. Notice that, in most cases, uniform grids are preferred to non-uniform grids, because they are easier to generate and the algorithms become simpler. However, in the case of adaptive refinement (see [34] for the case of isotropic materials) regions with irregular grids will naturally occur and therefore the above algorithm should be used in problems where adaptive refinement is employed for modeling the behavior of UD FRCs.

\section{Numerical results}

\subsection{Problems setup and computational details}

We consider the following setup for analyzing dynamic fracture phenomena in a UD composite lamina: a central-crack thin rectangular plate with $0.2 \mathrm{~m} \times 0.1 \mathrm{~m}$ as shown in Figure 5 . Two different loading types are employed in our simulations: in the first case A (Figure 6a) a uniform tensile load is applied suddenly along the left and right edges and maintained constant in time after that; in the second case B (Figure 6b), a uniform tensile load is applied suddenly on the crack surfaces and maintained constant in time after that. Both cases generate sharp stress wave (shock waves) but of different profiles and which interact differently with the boundaries and the crack surfaces. Dynamic experiments in UD FRC that produce the type of loading similar to case A (loading on the external boundaries of the sample), have been reported in [3]. Creating rapid loading on the crack faces is reported in the experiments in [39]. The composite material used in the examples shown below is the M55J/M18 carbon/epoxy [40]. The material properties are presented in Table 2.

\subsubsection{Computational details}

For the numerical spatial integration of the peridynamic equations (1) and (6) with the micromodulus functions as defined in Section 3, we use the mid-point integration as described in [35]. The computational results here are all based on uniform grids. In all examples in which the grid has a particular alignment with the fiber direction (like $45^{\circ}$ and $90^{\circ}$ ), the horizon size is $\delta=2 \mathrm{~mm}$ and the ratio between the horizon size and the grid spacing, the parameter $m$, is take here to be 5 . Reasons for these selections are given in [31] and are based on convergence tests for dynamic fracture in unidirectional fiberreinforced composites in which the fibers align with the computational grid. For the general case of arbitrary grid orientation relative to the fiber direction, we perform $m$-convergence and $\delta$-convergence tests.

We employ the explicit time integration Velocity-Verlet algorithm which is a more numerically stable version of centraldifferences [15, 41]. A uniform time step size of $50 \mathrm{~ns}$ is used for all simulations and this is a stable time step for the scheme used with the finest discretization employed in this work.

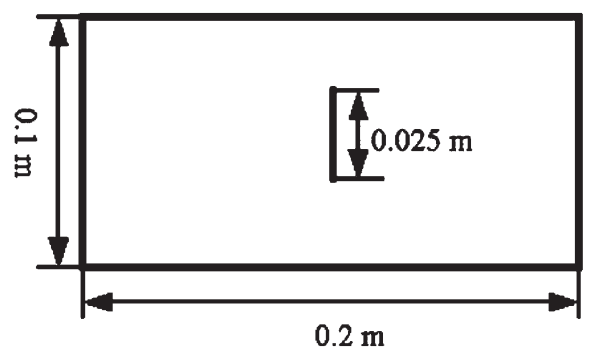

Figure 5. Geometry of the plate with a center notch for the dynamic tests on unidirectional fiber-reinforced composites. 

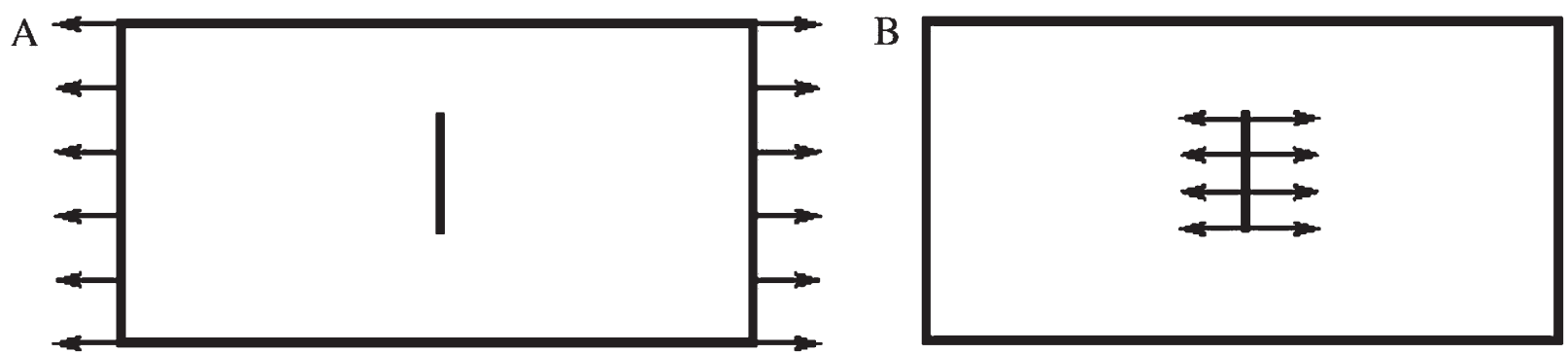

Figure 6. Two different loading cases. Case A: suddenly applied loading on the left and right boundaries; Case B: sudden loading on the pre-crack surfaces.

Table 2. Material properties.

\begin{tabular}{|c|c|}
\hline Property & Unidirectional \\
\hline Longitudinal Young's modulus $E_{11}(\mathrm{GPa})$ & 329 \\
\hline Transverse Young's modulus $E_{22}(\mathrm{GPa})$ & 6 \\
\hline Shear modulus $G_{12}(\mathrm{GPa})$ & 4.4 \\
\hline Poisson's ratio $v_{12}$ & 0.346 \\
\hline Density $\rho\left(\mathrm{kg} / \mathrm{m}^{3}\right)$ & 1630 \\
\hline Fracture energy $G_{0_{2}}^{11}\left(\mathrm{~kJ} / \mathrm{m}^{2}\right)$ & 15.49 \\
\hline Fracture energy $G_{0}^{\mathbb{2 2} 2}\left(\mathrm{~kJ} / \mathrm{m}^{2}\right)$ & 0.168 \\
\hline
\end{tabular}

All computations are performed in the original configuration (Lagrangian approach), so that searching for neighboring nodes is performed only once, at the beginning of the computations. For the short-range forces (penalty forces that prevent interpenetration of material) the relative distance in the current configuration is computed for nodes that are neighbors in the original configuration.

No damping of any kind is used in this study. The numerical examples shown below are obtained using an in-house 2D serial peridynamic code implemented in Fortran 90/95. All examples are run as serial jobs on a computer cluster with $2.2 \mathrm{GHz} / 64$ bit Opteron processors running Linux.

The $\delta=2 \mathrm{~mm}$ horizon and $m=5$ leads to a uniform discretization with $\Delta x=0.4 \mathrm{~mm}$, which is a total of 126,002 nodes. All peridynamic simulations below are performed with these values except for the convergence studies in the case of arbitrary grid orientation relative to the fiber direction. In [31] we showed that this nonlocal region gives crack patterns similar to those obtained with $\delta=1 \mathrm{~mm}$, and that the splitting mode crack propagation velocities with the $1 \mathrm{~mm}$ horizon are in the range of those obtained from analytical classical models for a delamination crack. In principle, for homogeneous materials, one needs to use a horizon sufficiently small relative to the geometrical features of the sample and the characteristics of the specific dynamic loading on the sample. If the material has a microstructure that leads to a particular material length scale, the horizon may be related to that (see also the discussion in [26]). From the efficiency point of view, a larger horizon leads to coarser grids and thus to faster solutions, but of course, a more "nonlocal" behavior and faster crack propagation speeds. A balance between good approximation of the observed behavior and computational efficiency has to be attained. The damage index of a node is utilized to monitor the fracture behavior in our peridynamic simulations. The damage index of a node is defined as the number of currently broken bonds by the initial number of bonds associated with that node $[15,31]$.

In all examples below, the uniform discretization grid is aligned with the horizontal and vertical axes.

\subsection{Dynamic fracture and damage simulations for the case when the angle between the grid and the fiber orientation is $45^{\circ}$}

In this section, we investigate the damage pattern and pro- gression for the $45^{\circ}$ fiber orientations by using the model derived semi-analytically in Section 3.1. Two different loading cases are employed in order to investigate how the stress waves affect the evolution of the damage process and crack propagation.

\subsubsection{Damage behavior and crack patterns for the loading Case A}

For this loading case, a "no-fail zone" is used on the boundary nodes where the loads are suddenly applied, in order to prevent rupture at those locations [31]. The uniform tensile loading $\sigma=6 \mathrm{MPa}$ is applied abruptly along the left and right boundary for $\varphi=45^{\circ}$. The result showing the damage map for $\varphi=45^{\circ}$ is given in Figure 7. In all the damage maps below we use the same range for the color-bar of the damage index as in Figure 7.

In all the simulations, the splitting fracture mode is observed without fiber breakage. Interestingly, extensive and diffuse damage in the matrix, beside the splitting mode from the tips of the pre-crack are obtained for $\varphi=45^{\circ}$, are seen in Figure 7. In Figures 8a-c and 9, we show a few snapshots of the time evolution of damage maps and strain energy profiles for $\varphi=45^{\circ}$. As the shock waves propagate in the anisotropic material and meet at the center pre-crack, the splitting mode combines with diffuse matrix cracking (see Supplementary Video 3). In fact, when the main wave-front moving through the matrix reaches the center pre-crack, matrix cracking is initiated and extensively propagates instead of the splitting mode, between $100 \mu$ s and $200 \mu$ s (see Supplementary Video 1). The matrix in the composite is completely shattered in those regions. Eventually, splitting along the fiber directions starts to progress at about $350 \mu \mathrm{s}$ and full separation of the composite into two pieces follows soon after. Figure $8 \mathrm{~d}$ shows that, at these load levels, the "fiber bonds" are not damaged, except for those cut by the pre-crack. Damage of "fiber bonds" is computed by only counting the broken "fiber bonds" and the original number of such bonds. We notice that for $\varphi=45^{\circ}$, the damage behavior under dynamic loading is more complex than under static loading which only contains the splitting mode [4]. This mixing of extensive matrix shattering followed by complete splitting is observed even if we lower the

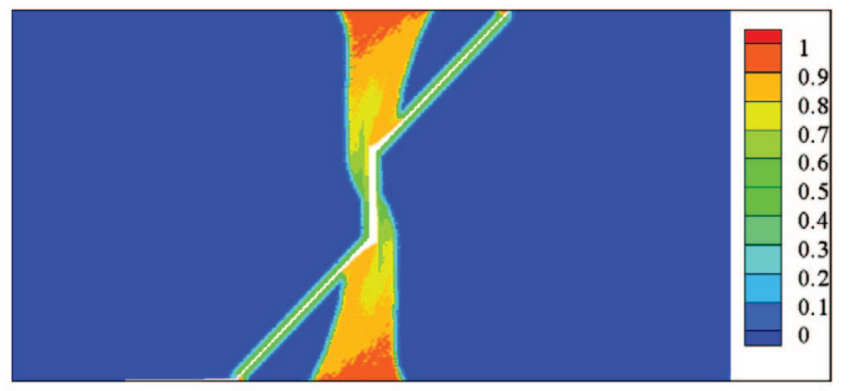

Figure 7. Damage map at $500 \mu$ s for the $45^{\circ}$ fiber orientation under loading Case A. 


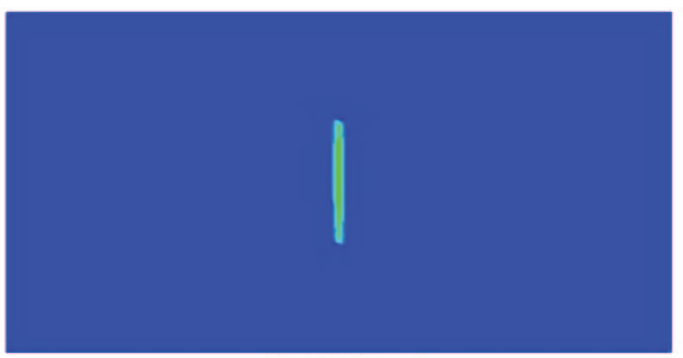

(a) $[100 \mu \mathrm{s}]$

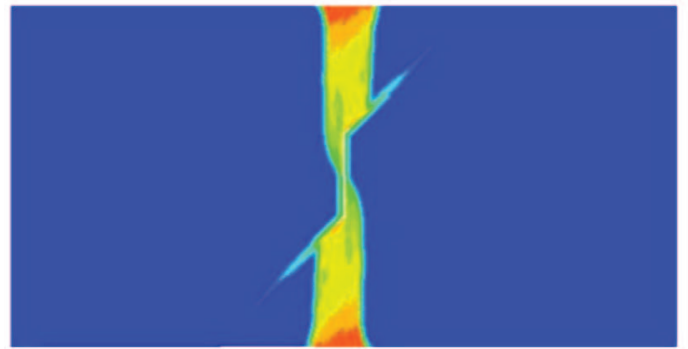

(c) $[350 \mu \mathrm{s}]$

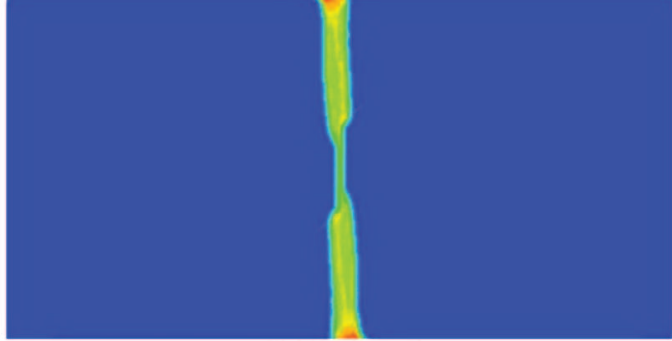

(b) $[200 \mu \mathrm{s}]$

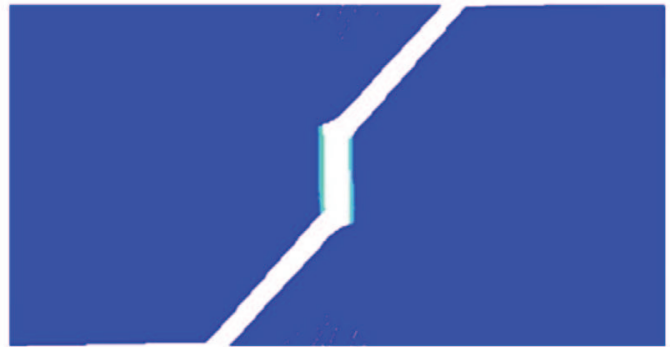

(d) fiber-damage map

Figure 8. Time-evolution of damage for $\varphi=45^{\circ}$ : (a) matrix cracking (at $100 \mu \mathrm{s}$ ); (b) extensive matrix cracking and diffuse damage (at $200 \mu \mathrm{s}$ ); (c) extensive diffuse damage and growth of splitting mode fracture (at $350 \mu \mathrm{s})$; (d) damage map for the "fiber bonds" only (at end of the simulation when total separation due to splitting is clearly visible).

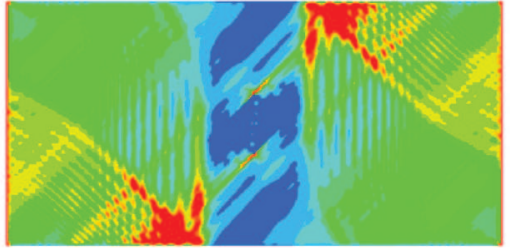

$[75 \mu \mathrm{s}]$

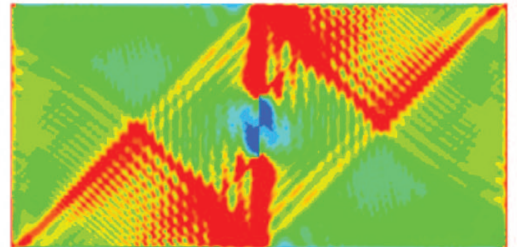

$[95 \mu \mathrm{s}]$

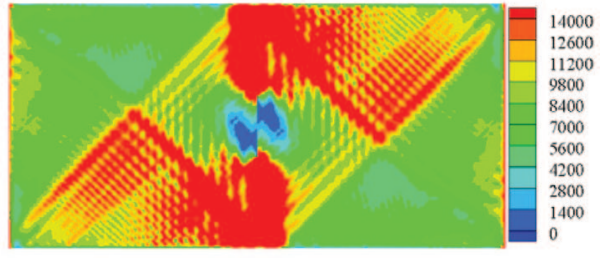

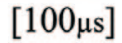

Figure 9. Elastic strain energy profiles just before and after the pre-crack starts propagation.

magnitude of the dynamically applied tensile loading. In the elastic strain energy density figures (see Figure 9), some of the ripples behind the wave fronts are induced by the non-local region (horizon) size and some are a result of the numerical dispersion of the sharp wave. The numerical dispersion can be eliminated by using flux-corrected transport (FCT) algorithms [42].

\subsubsection{Evolution of fracture and crack patterns for the loading Case B}

An experimental device capable of suddenly generating uniform pressure along pre-crack surfaces was introduced in [39]. Such dynamic loading is similar to that shown in loading Case B (see Figure 6). A uniform pressure loading $\sigma=10 \mathrm{MPa}$ is applied abruptly along the pre-crack faces for the case when $\varphi=45^{\circ}$. The results showing the damage map for are given in Figure 10.

The damage pattern is changed significantly compared with the loading Case A, for this fiber-grid orientation angle. This is seen from comparing Figure 10 with Figure 7. Under Case B loading conditions, the diffuse matrix cracking is absent and is replaced by a distinct crack growth in the matrix that starts off as splitting fracture but it progresses by bending and thus migrating over the "fiber bonds" until it finally arrests in an almost vertical direction. A close examination of the elastic strain energy density evolution as the crack propagates, shows that the reflected stress waves from the boundaries influence the running crack and induce the bending, migration, and the ultimate arrest of the crack.

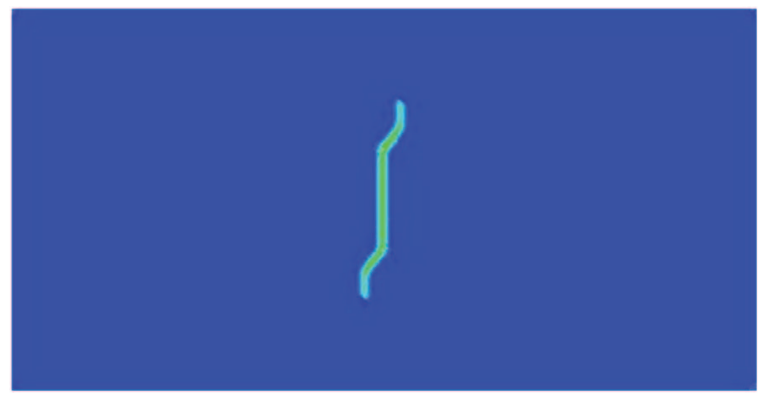

Figure 10. Damage map for $45^{\circ}$ fiber orientation under loading Case B.

On the top row of Figure 11, the elastic strain energy is plotted at three different times to illustrate the elastic wave moving away from the pre-crack faces, the reflected wave from the boundaries, and the interaction of the reflected wave with the crack tip. On the bottom row of Figure 11, damage maps show the crack propagation in time. The crack starts to propagate along the $45^{\circ}$ fiber orientation, in splitting mode, but immediately bends and "migrates" over "fiber bonds" without breaking any of them. The stress wave generated by the suddenly applied pressure on the crack faces moves toward to the boundaries and reflects from them at about $91 \mu$ s. The particular angles at which the reflected wave meets the crack tip results in curving of the crack path. It is interesting to observe that, due to the anisotropy and the asymmetry of the fiber ori- 


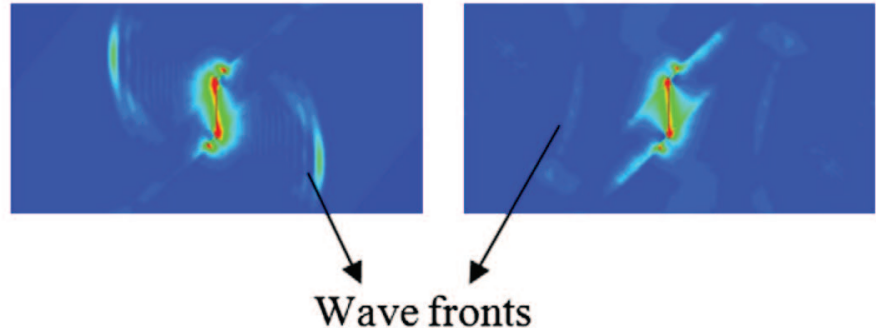

$[49 \mu \mathrm{s}]$

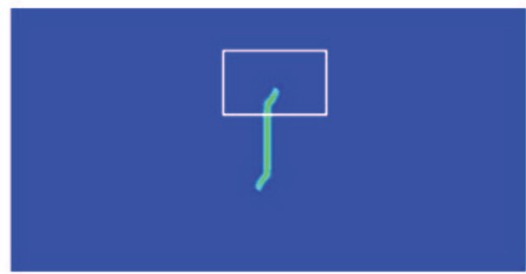

$[171.5 \mu \mathrm{s}]$
$[140 \mu \mathrm{s}]$

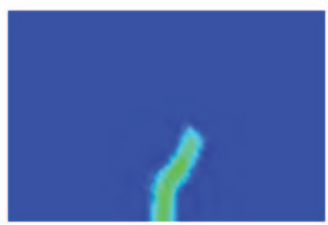

$[175 \mu \mathrm{s}]$

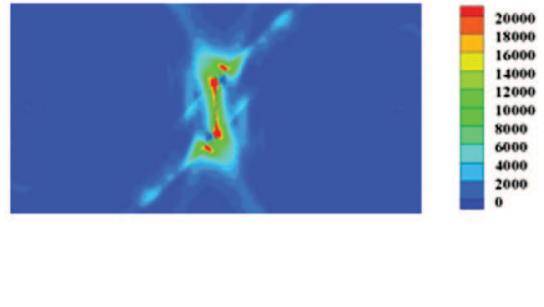

$[171.5 \mu \mathrm{s}]$

Figure 11. Elastic strain energy profiles (top row of figures) and damage maps around the crack tip area (bottom row of plots) for the $45^{\circ}$ fiber orientation under loading Case B (loading of the crack faces).

entation relative to the sample geometry, the reflected waves from the right and left boundaries reach the top crack tip at different times (171.5 $\mu$ s and $178.5 \mu \mathrm{s}$, respectively) and at different angles. Due to this, the interaction with the stress waves changes the crack growth direction. The role of the dynamic interaction between a crack and a stress wave has been experimentally studied in isotropic materials [39].

In the next section we study the effect of applying higher amplitude loadings.

\subsection{The effect of the loading magnitude on the dynamic frac- ture behavior}

In this section, we perform peridynamic simulations for $\varphi=45^{\circ}$ and $\varphi=90^{\circ}$ under different dynamic loading amplitudes. For the $45^{\circ}$ test we only report results with the loading Case B (on the crack surfaces), since in the other case the character of the damage does not change by further increasing the loading (in the range of loads we have tested). We apply the sudden pressure along the crack surface with two different magnitudes $\sigma=20 \mathrm{MPa}$ and $\sigma=33 \mathrm{MPa}$. In Figure 12, we observe that the splitting mode is dominant and the crack advances substantially before the reflected elastic waves reach the growing crack. As seen from Figure 12a, the splitting fracture transitions in the matrix fracture at the point when the crack is beginning to bend. When the loading is sufficiently strong, the splitting crack runs through and the stress waves do not reach the moving crack in time to cause a fracture mode change (see Figure 12b).

We now turn to the $90^{\circ}$ fiber orientation and analyze the dynamic fracture modes under different loading magnitudes.

For the $90^{\circ}$ fiber orientation case, only the splitting mode is observed in both loading cases, when the loading is $\sigma=2 \mathrm{MPa}$ in Case $\mathrm{A}$, and $\sigma=8 \mathrm{MPa}$ in Case B (see Figure 13a and c). In contrast with what happens in the $45^{\circ}$ case in loading Case B, for the $90^{\circ}$ fiber orientation case the reflected wave reaches the crack tip at the same time and having the same incidence angle. The crack path, therefore, is straight and only the splitting mode is observed for this level of loading. When we increase the loading amplitudes, however, from $2 \mathrm{MPa}$ to $4 \mathrm{MPa}$ in Case $\mathrm{A}$, and from $8 \mathrm{MPa}$ to $12 \mathrm{MPa}$ in Case $\mathrm{B}$, we observe dramatic differences compared to the lower amplitude loadings (see Figure $13 \mathrm{~b}$ and $\mathrm{d}$ ). In both loading Cases $\mathrm{A}$ and $\mathrm{B}$, after an initial splitting crack growth from the center pre-crack, the cracks branch! This indicates a fracture mode change, from splitting to cracks migrating through the matrix over the "fiber bonds." We observe no "fiber bonds" damage at these dynamic loading levels. The branching angles are around $45^{\circ}$. Crack branching in dynamic fracture in brittle materials has been experimentally studied in glass plates [10] and peridynamics has been shown to be able to predict this behavior $[15,16]$. To obtain this phenomenon in the anisotropic UD FRCs was a surprise to us. The recent dynamic experiments in [3], however, demonstrate

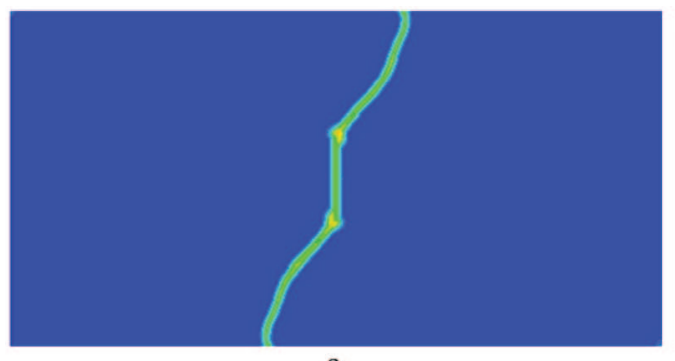

a

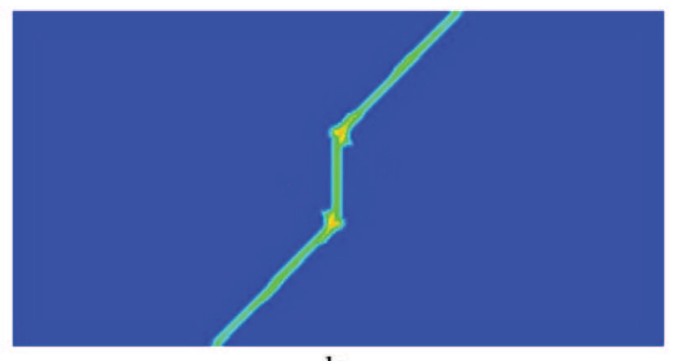

b

Figure 12. Damage patterns for $45^{\circ}$ fiber orientation when the loading magnitudes (Case B, crack face loading) are increased to $\sigma=20 \mathrm{MPa}$ (left) and $\sigma=33 \mathrm{MPa}$ (right). 

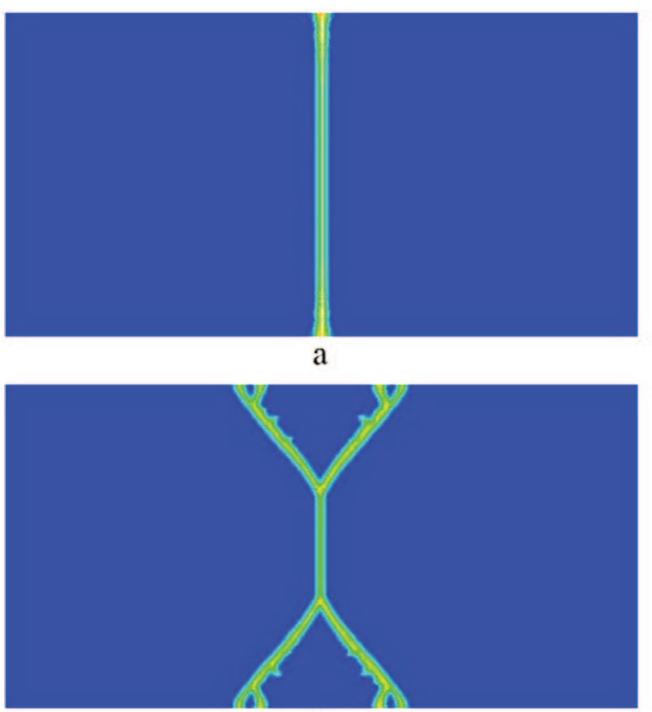

c

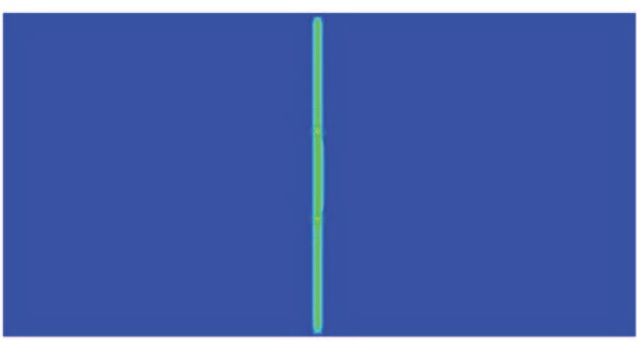

b

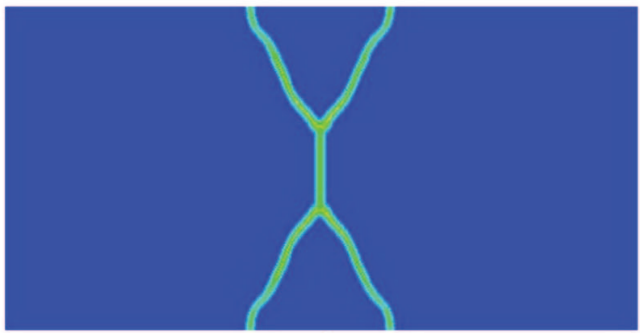

d

Figure 13. Crack patterns for the case $\varphi=90^{\circ}$ under different loading magnitudes: loading Case A with (a) $\sigma=2 \mathrm{MPa}$, and (b) $\sigma=4 \mathrm{MPa}$; and loading Case B with (c) $\sigma=8 \mathrm{MPa}$, and (d) $\sigma=12 \mathrm{MPa}$.

that this phenomenon actually happens in reality in dynamically loaded unidirectional FRCs. As in our simulations, the experiments indicate no breakage of fibers in these branching cracks, meaning that the cracks grow in the matrix migrating over the fibers. To our knowledge, this is the first computational prediction for this phenomenon. The experimental branching angle, for an experiment in which the specimen is dynamically loaded from its boundaries, was around $45^{\circ}$.

The peridynamic results also indicate that secondary or multiple branching can happen (see Figure 13b, and Supplementary Videos 2 and 4) and that interaction between the propagating cracks and the stress waves induce some crackpath bending (Figure 13d).

The relation between the stress waves and crack curving can be seen from the sequence of plots taken at different times for the loading Case B when the load magnitude is $12 \mathrm{MPa}$, shown in Figure 14. The top plots of Figure 14 indicate that the reflected waves move towards the center of the plate and meet the branching crack tips at $145 \mu$ s. The branches were propagating straight before that. After the reflected waves hit the branch tips, the cracks start to curve as seen from the bottom plots of Figure 14.

\subsection{Dynamic fracture and damage simulations for an arbitrary grid orientation relative to the fibers using the proposed algorithm}

For an arbitrary grid orientation relative to the fiber orientation in the composite lamina, the proposed Algorithm 1 is employed to compute the scaling factors for "fiber bonds" and "matrix bonds." We use two different grids for the same horizon size ( $m$-convergence study) and also perform a $\delta$-convergence study. Of interest are the damage patterns and evolution, as well as the development and propagation of splitting cracks. Because of this, we select the loading Case A, since this case, as we have seen above, results in a very interesting combination of diffuse-type damage ("matrix shattering") followed by isolated splitting cracks. Thus, along the left and right boundaries, a sudden tensile loading $\sigma=6.75 \mathrm{MPa}$ is applied. We use a higher magnitude loading than that in Section 4.2 in

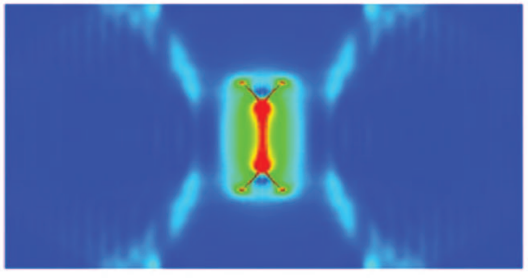

$[130 \mu \mathrm{s}]$

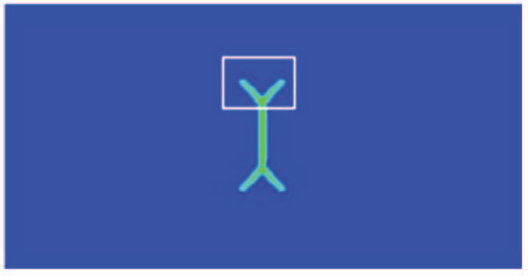

[145 $\mu \mathrm{s}]$

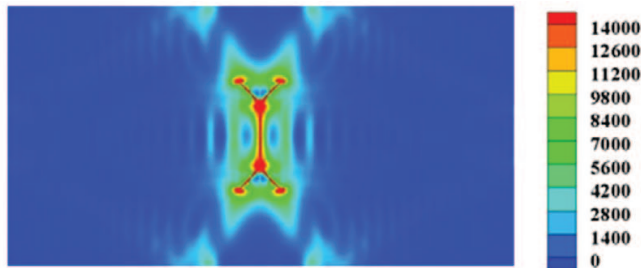

$[145 \mu \mathrm{s}]$

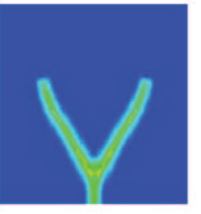

$[155 \mu \mathrm{s}]$

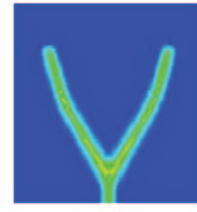

$[165 \mu \mathrm{s}]$

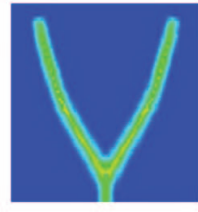

[175 $\mu \mathrm{s}]$

Figure 14. Elastic strain energy profiles (top row) and damage maps around the crack tips (bottom row) at different times for loading Case B and $\sigma=12 \mathrm{MPa}$. 


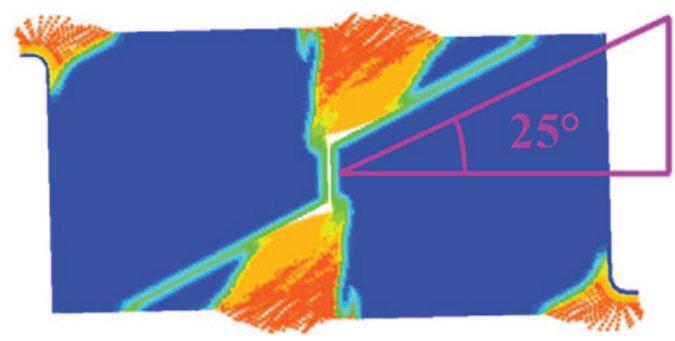

a

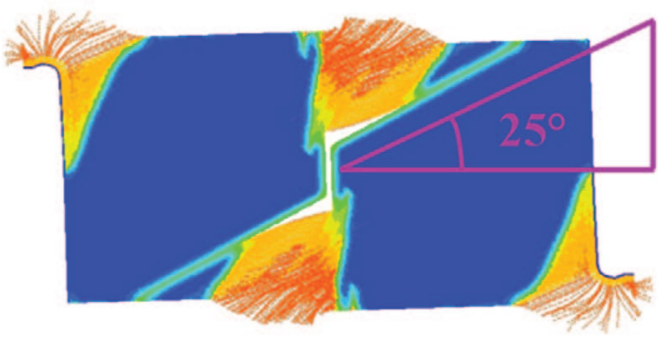

b

Figure 15. Damage maps for $\varphi=25^{\circ}$ with different $m$. (a) $m=5$ at $75.2 \mu$ s; (b) $m=7$ at $78.2 \mu \mathrm{s}$.

order to better observe the damage behavior in the "shattered" region. Given the dynamic nature of the loading, in which wave reflections and deflections from the boundaries and newly formed crack surface dramatically influence the solution, these are extremely tough tests to pass by any numerical method.

We choose the case in which the angle between the grid and the fiber orientation is $\varphi=25^{\circ}$. Given the results obtained in [31], for the $m$-convergence tests we select only two values for the $m$ parameter: $m=5$ and $m=7$, and a fixed horizon size $\delta=4 \mathrm{~mm}$. Recall that the angle $a$ that provides the cone within which we assign fiber bonds (see Algorithm 1) should be, in principle, connected to the $m$ value. For simplicity here we use $a=3^{\circ}$ for both $m$ values. As shown in Figure 15 , we observe diffuse damage in the matrix and splitting fracture mode in both cases. Movies 6 and 7 in the supplementary data show the evolution of damage for $m=5$ and $m=7$, respectively, while movies 9 and 10 depict the strain energy density evolution for $m=5$ and $m=7$, respectively. Furthermore, the angles for the major splitting crack are the same in both cases, and very close to the fiber orientation angle of $25^{\circ}$. Since the nodes picked up by Algorithm 1 that are designated to have "fiber bonds" with the central node are obviously different between $m=5$ and $m=7$ (see Figure 4), we conclude that $m$-convergence is achieved. In addition, since these nodes are not aligned in any special way (compare with the 0,45 , or 90 orientations) the results also demonstrate that the algorithm will perform the same even when a random, non-uniform discretization is used. Of course, under such a discretization the

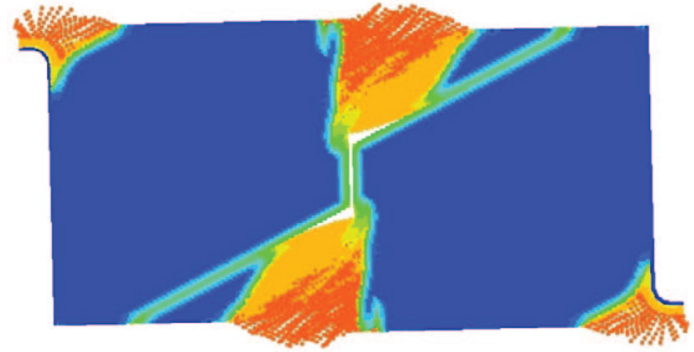

a

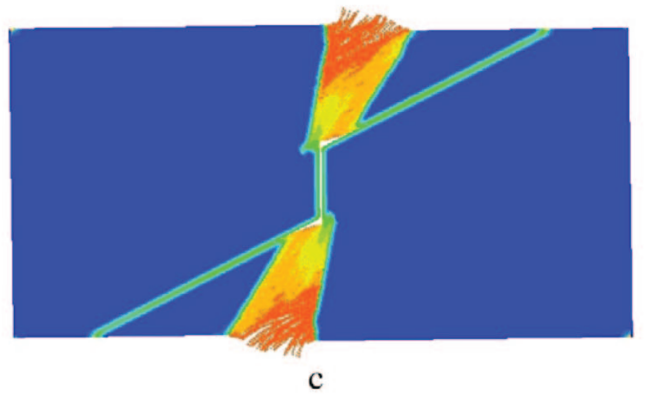

methods introduced in [32] should be used in the computation of the peridynamic forces.

The large magnitude sudden loading sends shock waves that bounce off the boundaries which creates extra damage zones near the corners of the samples. The no-fail conditions enforced on the peridynamic bonds near the left and right boundaries are now visible. While the shattered matrix region and the splitting crack are almost identical between the two different grids (which use the same horizon size) and obtained at about the same time, the corner-damage is more extensive in the finer grid than in the coarser grid. This can be explained by how slightly different wave dispersions between the grids interact with the peridynamic bonds. The "matrix bonds" are shattered in the central diffuse-damage region as well as in the corner regions and the "fiber bonds" in these areas become separated. Observe that when $m=7$ (denser grid) there are more loose "fiber bonds" than in the coarser model with $m=5$. Interestingly, under the given loading, no "fiber bonds" suffer damage.

In what follows we select $m=5$ to perform a $\delta$-convergence test for the fiber orientation $\varphi=25^{\circ}$. The results will indicate which horizon size is sufficiently small to capture the main features of the damage evolution and for which using a smaller horizon size would not result in qualitatively different results. From a practical point of view, one wants to use a larger horizon size to reduce the computational burden. We use the following horizon sizes and the resulting discretizations from a value $m=5: \delta=4 \mathrm{~mm}$ with $\Delta x=0.8 \mathrm{~mm}$ (31,752 nodes), $\delta=3 \mathrm{~mm}$ with $\Delta x=0.6 \mathrm{~mm}$ (56,112 nodes), and

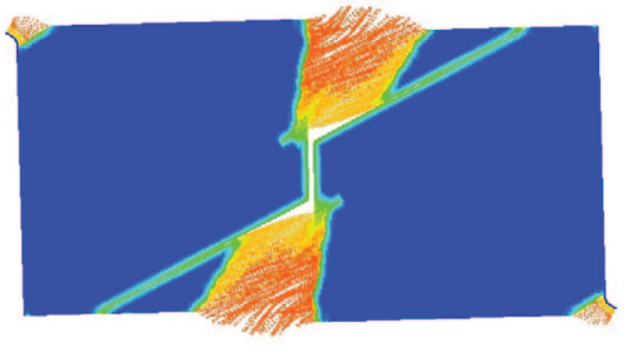

b

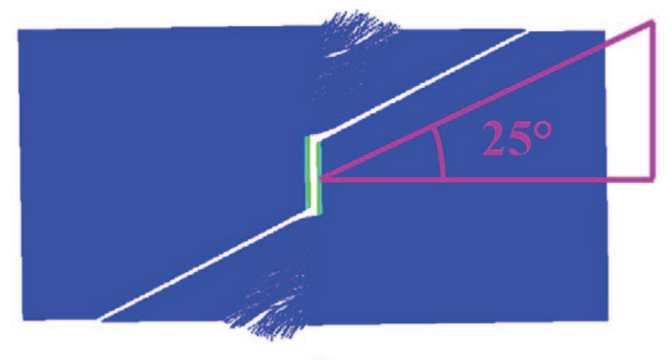

d

Figure 16. Damage maps for $\varphi=25^{\circ}$ at different horizon size. (a) $\delta=4 \mathrm{~mm}$ at $75.2 \mu \mathrm{s}$; (b) $\delta=3 \mathrm{~mm}$ at $90 \mu \mathrm{s}$; (c) $\delta=2 \mathrm{~mm}$ at $135 \mu \mathrm{s}$; (d) The damage map for "fiber bonds" only. 

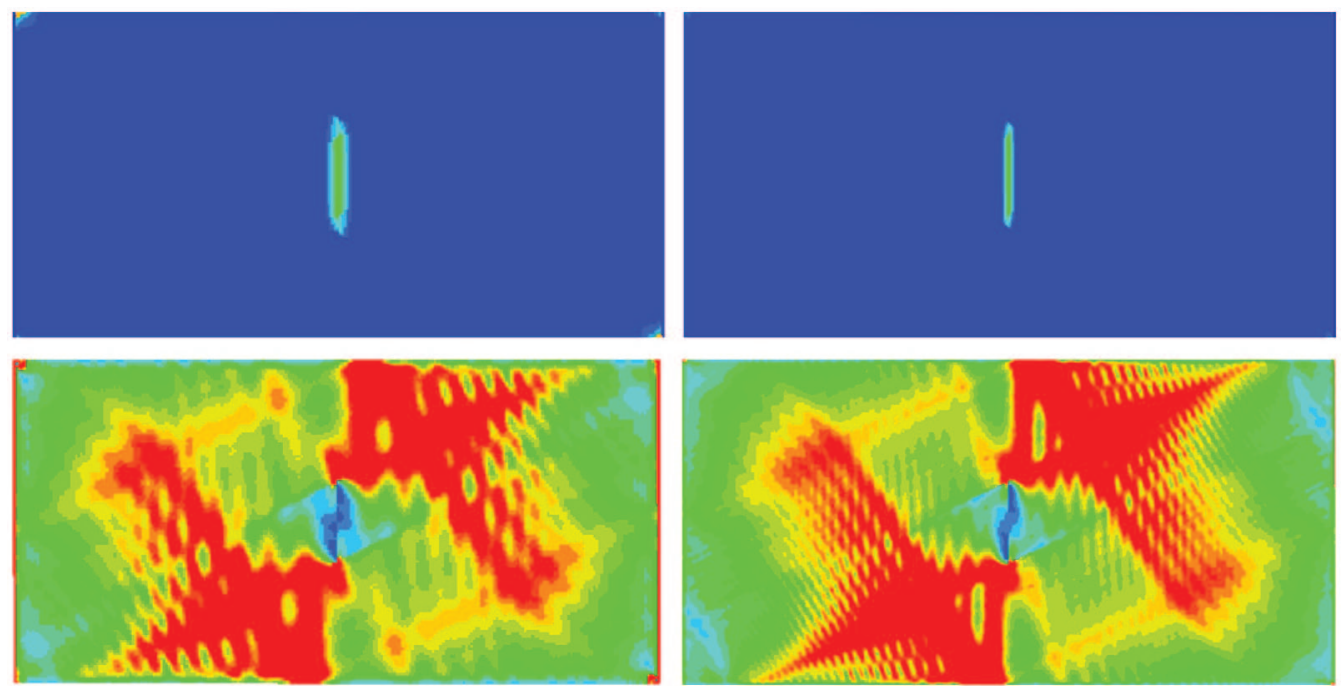

Figure 17. Damage maps (top) and strain energy density plots (bottom) for $\delta=4 \mathrm{~mm}$ (left) and $\delta=2 \mathrm{~mm}$ (right) at about $10.5 \mu$.

$\delta=2 \mathrm{~mm}$ with $\Delta x=0.4 \mathrm{~mm}(126,002$ nodes). The same loading conditions are used as in the $m$-convergence study above.

The results in terms of the damage maps are shown in Figure 16 and in movie 6 for $\delta=4 \mathrm{~mm}$, and movie for 5 for $\delta=2 \mathrm{~mm}$. As shown in Figure 16a-c, for different horizon sizes, the similar damage patterns are observed, such as diffuse damage in the "matrix bonds" (shattered matrix) followed (see below for the time-evolution of damage) by the splitting fracture mode. Notice the change in size of the damaged area produced by the meeting of the stress waves in the center, and the subsequent interactions between stress waves reflected from the boundaries (see movies 9 and 8 with the strain energy density for $\delta=4 \mathrm{~mm}$, and $\delta=2 \mathrm{~mm}$, respectively). The reason for the larger damage areas when the horizon is larger is discussed in detail in [15]. As shown in Figure $16 \mathrm{~d}$, we do not observe any damage of the "fiber bonds", other than that generated by the initial center cut. The measured angle of the splitting crack is very close to the fiber orientation $\varphi=25^{\circ}$, and is about $26^{\circ}$. Moreover, in the shattered-matrix region, we see ends of "fibers" becoming "loose." This happens because all the "matrix bonds" for nodes in those regions have been broken. We also observe that the damage around the corner areas is decreasing as the horizon decreases. This damage is produced initially by the abrupt loading, especially with the larger horizon size, and its growth is induced at later times by the stress waves moving through the material. Notice that the times at which the damage maps look similar between the different horizon sizes are significantly different. This is due to the different time-evolution of damage and propagation of splitting cracks obtained when different nonlocal regions (horizon sizes) are used. This behavior is induced by two factors, both related to the horizon size:

1. The wave dispersion of stress waves depends on the horizon size (see [14]), and

2. The "thickness" of the damage zone is related to the horizon size (see [15]).

In what follows we discuss in detail the time-evolution of damage and how the two factors mentioned above play a role in influencing when the splitting cracks start propagating and how fast they grow. The damage maps and elastic strain energy plots for both $\delta=4 \mathrm{~mm}$ and $\delta=2 \mathrm{~mm}$ at different times are shown in Figure 17, Figure 18, and Figure 19. The damage of "matrix bonds" in the central region happens immediately after the shock waves arriving from the left and right boundaries reach the pre-crack at about $10.5 \mu$ s (see Figure 17 and Supplementary Videos 6 and 9 for $\delta=4 \mathrm{~mm}$ and 5 and 8 for $\delta=2 \mathrm{~mm}$ ). The damage pattern and strain energy profiles for
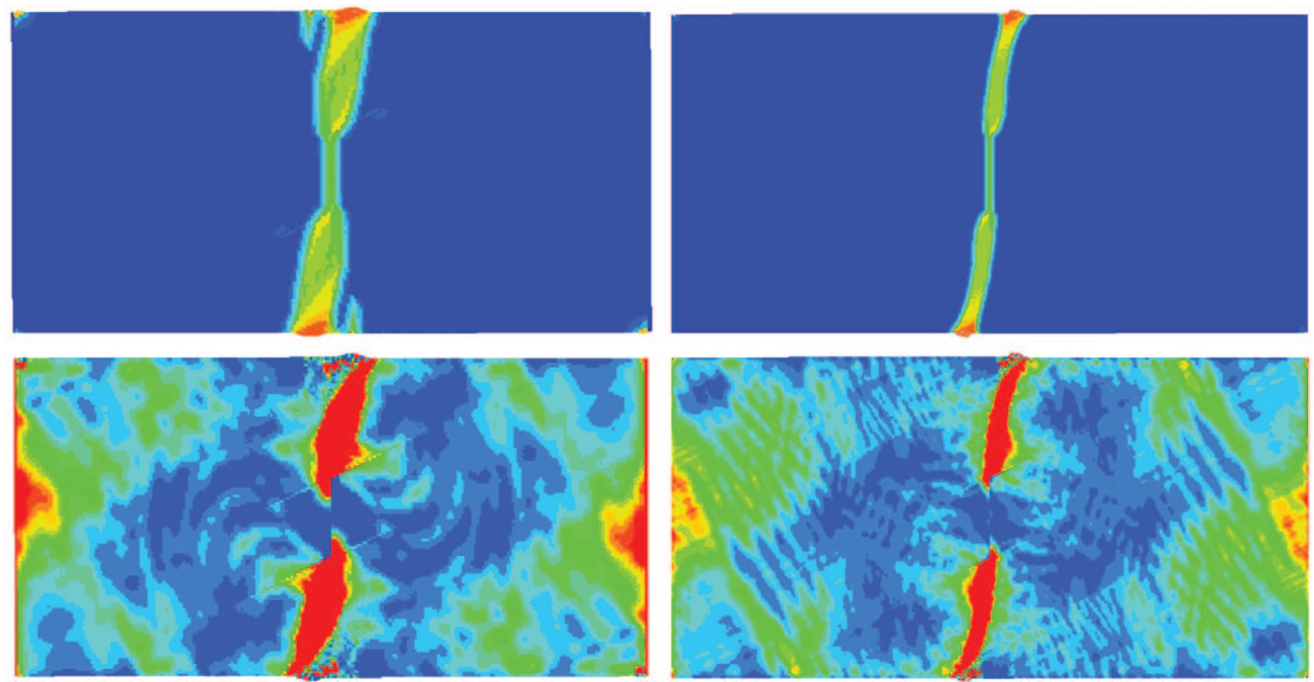

Figure 18. Damage maps (top) and strain energy density plots (bottom) for $\delta=4 \mathrm{~mm}$ (left) and $\delta=2 \mathrm{~mm}$ (right) at about $24 \mu \mathrm{s}$. 

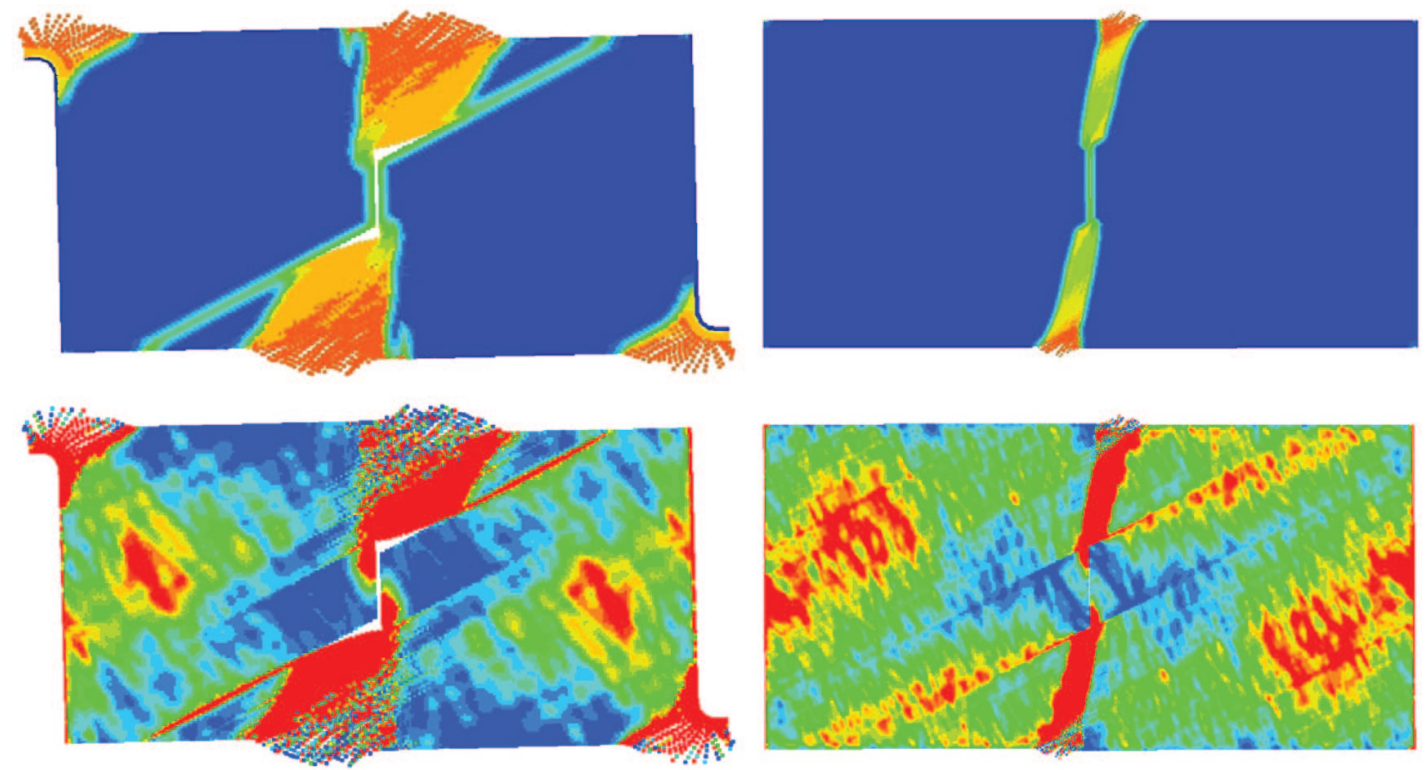

Figure 19. Damage maps (top) and strain energy density plots (bottom) for $\delta=4 \mathrm{~mm}$ (left) and $\delta=2 \mathrm{~mm}$ (right) at about $75.2 \mu \mathrm{s}$.

two different horizon sizes are approximately the same at this time. However, the oscillations behind the shock front, which are caused by the wave dispersion induced by nonlocality, become smaller with a decreasing horizon size. At about $24 \mu \mathrm{s}$, the damage patterns are about the same but the stress waves start showing some more pronounced differences, especially near the damage region, as seen from the strain energy density maps in Figure 18. The horizon size influences the effective "thickness" of the damage zone (see [15]) and we start to observe a thicker shattered matrix region. That, in turn, significantly influences the reflection and deflection of the stress waves that continue to damage the composite as they travel through the sample.

We roughly estimate the average crack propagation speed for the splitting crack and obtain that is about $5600 \mathrm{~m} / \mathrm{s}$ for $\delta=4 \mathrm{~mm}$ and about $3400 \mathrm{~m} / \mathrm{s}$ for $\delta=2 \mathrm{~mm}$. While the longitudinal waves speed is the same independent of the horizon size (the micromodulus functions are obtained so that the microelastic material has the same elastic modulus as a classical material) the crack propagation speeds are lower for the smaller horizon size (see, e.g. [15, 16]). We explain this as follows: the trailing waves behind the shock front (induced by both the nonlocal region size and the numerical discretization and modified by reflections from boundaries) can strongly influence the crack propagation speed, by speeding it up or slow it down. We observed the same behavior in the case with $0^{\circ}$ fiber orientation (see [31]). It is important to notice that when the loading is so that, at least for a while, the crack propagation is not influenced by stress waves, the crack propagation speed does not depend on the horizon size. This is demonstrated in [26] by applying sudden loadings on the faces of a pre-crack.

\section{Conclusions}

We introduced a new computational model of the homogenized peridynamic formulation for unidirectional (UD) fiberreinforced composites (FRCs). The model can be used with any type of uniform or non-uniform discretization, and for any fiber orientation relative to the grid orientation. We used the model to simulate dynamic fracture and damage in $45^{\circ}, 90^{\circ}$, and $25^{\circ}$ degree laminas. For the special case when the angle between the fibers and a uniform grid is $45^{\circ}$ we derived, semianalytically, the scaling factors in the model. For the general case, we introduced an algorithm that correctly scales the discrete model so that the computed strain energy density in the composite is independent of the grid spacing or the grid orientation relative to the fiber direction. We performed convergence studies for the arbitrary grid orientation in terms of grid refinement for a fixed horizon size and in terms of decreasing the horizon size. The propagation of the splitting crack type was shown to match the angle of the fiber orientation.

The detailed investigations with respect to the location of the suddenly applied loadings (on the outer boundaries or on the center pre-crack faces) and with respect to the magnitude of the suddenly applied loads led to the following conclusions:

- The failure and damage patterns induced by dynamically loading a UD FRC composite are strain rate dependent and dramatically more complex than what is observed from quasi-static loadings. No explicit rate-dependence was used in the peridynamic model. The inertia and wave propagation led directly to the observed behavior.

- The stress waves control the dynamic crack propagation process, influencing curving of crack paths and crack arrest, as well as the crack propagation speed.

- The dynamic effects lead to coexistence of damage modes and to transitions between these modes. Depending on the type of loading (from the boundaries, or from the interior pre-crack faces) the peridynamic model captures splitting fracture, diffuse damage (shattering) in the matrix and separation between "fiber bonds," crack curving, and crack migration in the matrix, and matrix crack branching.

- The dynamic fracture and damage profiles obtained from the peridynamic model are consistent with recent experimental observations on dynamic fracture in UD FRCs. For example, branching of a splitting crack into two matrix cracks is observed in UD composites dynamically loaded along the transverse direction. Branching does not happen unless the load level is sufficiently high. In both the experiments and the peridynamics computations, this type of loading does not cause breakage of the fibers (or fiber bonds).

No special criteria for splitting fracture, for crack curving or crack branching, or for transitions between fracture modes were used. The only inputs in our peridynamic model were the elastic moduli for the anisotropic material and the longitu- 
dinal and transverse mode I fracture energies. The nonlocal region (the peridynamic horizon) was selected so that it would be sufficiently small relative to the geometric and dynamic conditions in order to efficiently obtain results deemed close to converged ones.

Acknowledgments - The authors are thankful for the financial support offered through research contracts between UNL and the ARO (Dr. Larry Russell), and ARL (project coordinators Dr. C.F. Yen and Dr. C. Randow), ARO award number 58450EG. The authors would like to thank the two anonymous reviewers whose comments led to many improvements in the manuscript.

\section{Supplementary data}

Ten supplementary videos with the simulation results described in this paper are linked to the repository cover page for this article.

\section{References}

[1] H. Jeff, The Boeing 787 dreamliner: More than an airplane, Presentation to AIAA/AAAF Aircraft Noise and Emissions Reduction Symposium, American Institute of Aeronautics and Astronautics and Association Aéronautique et Astronautique de France, Monterey, California, 2007.

[2] S. Kazemahvazi, D. Zenkert, M. Burman, Notch and strain rate sensitivity of non-crimp fabric composites, Compos. Sci. Technol. 69 (2009) 793-800.

[3] A. Haque, M. Ali, High strain rate responses and failure analysis in polymer matrix composites - An experimental and finite element study, J. Compos. Mater. 39 (2005) 423-450.

[4] E. M. Wu, Fracture mechanics of anisotropic plates, in: Compos. Mater. Workshop, Technomic Publishing, Lancaster, PA, 1968, pp. $20-43$.

[5] S. C. Tan, Mixed-mode fracture of notched unidirectional and offaxis laminates under tensile loading, J. Compos. Mater. 23 (1989) 1082-1105.

[6] G. F. Sun, Fracture of fiberglass reinforced composites, J. Compos. Mater. 15 (1981) 521-530.

[7] K. Tohgo, S. D. Wang, T. W. Chou, A criterion for splitting crack initiation in unidirectional fiber-reinforced composites, J. Compos. Mater. 27 (1993) 1054-1076.

[8] S. R. Hallett, B. G. Green, W. G. Jiang, M. R. Wisnom, An experimental and numerical investigation into the damage mechanisms in notched composites, Composites Part A - Appl. Sci. Manuf. 40 (2009) 613-624.

[9] I. M. Daniel, J. J. Luo, P. M. Schubel, B. T. Werner, Interfiber/interlaminar failure of composites under multi-axial states of stress, Compos. Sci. Technol. 69 (2009) 764-771.

[10] F. P. Bowden, J. H. Brunton, J. E. Field, A. D. Heyes, Controlled fracture of brittle solids and interruption of electrical current, Nature 216 (1967) 38-42.

[11] E. J. Pineda, A. M. Waas, B. A. Bednarcyk, C. S. Collier, P. W. Yarrington, Progressive damage and failure modeling in notched laminated fiber reinforced composites, Int. J. Fract. 158 (2009) 125143.

[12] T. E. Tay, G. Liu, V.B.C. Tan, X. C. Sun, D. C. Pham, Progressive failure analysis of composite, J. Compos. Mater. 42 (2008) 19211966.

[13] J. M. Guimard, O. Allix, N. Pechnik, T. Pascal, Characterization and modeling of rate effects in the dynamic propagation of modeII delamination in composite laminates, Int. J. Fract. 160 (2009) 5577.

[14] S. A. Silling, Reformulation of elasticity theory for discontinuities and long-range forces, J. Mech. Phys. Solids 48 (2000) 175-209.

[15] Y. D. Ha, F. Bobaru, Studies of dynamic crack propagation and crack branching with peridynamics, Int. J. Fract. 162 (2010) 229244.

[16] Y. D. Ha, F. Bobaru, Characteristics of dynamic brittle fracture captured with peridynamics, Eng. Fract. Mech. 78 (2011) 11561168.
[17] J. T. Foster, S. A. Silling, W. Chen, An energy based failure criterion for use with peridynamic states, Int. J. Multiscale Comput. Eng. 9 (2011) 675-688.

[18] A. C. Eringen, D.G.B. Edelen, Nonlocal elasticity, Int. J. Eng. Sci. 10 (1972) 233-248.

[19] I. A. Kunin, Elastic Media with Microstructure I: One-dimensional Models, Springer Series in Solid-State Sciences, vol. 44, Springer, Berlin, Heidelberg, New York, 1982.

[20] I. A. Kunin, Elastic Media with Microstructure II: Three-dimensional Models, Springer Series in Solid-State Sciences, vol. 44, Springer, Berlin, Heidelberg, New York, 1983.

[21] H. Gao, P. Klein, Numerical simulation of crack growth in an isotropic solid with randomized internal cohesive bonds, J. Mech. Phys. Solids 46 (1998) 187-218.

[22] P. Klein, H. Gao, Crack nucleation and growth as strain localization in a virtual-bond continuum, Eng. Fract. Mech. 61 (1998) 21-48.

[23] P. A. Klein, J. W. Foulk, E. P. Chen, S. A. Wimmer, H. J. Gao, Physics-based modeling of brittle fracture: Cohesive formulations and the application of meshfree methods, Theor. Appl. Fract. Mech. 37 (2001) 99-166.

[24] Z. Zhang, X. Ge, Micromechanical modelling of elastic continuum with virtual multi-dimensional internal bonds, Int. J. Numer. Meth. Engrg. 65 (2006) 135-146.

[25] S. A. Silling, O. Weckner, E. Askari, F. Bobaru, Crack nucleation in a peridynamic solid, Int. J. Fract. 162 (2010) 219-227.

[26] F. Bobaru, W. Hu, The meaning, selection, and use of the peridynamic horizon, Int. J. Fract., 176 (2012), 215-222; doi: 10.1007/ s10704-012-9725-Z

[27] J. Xu, E. Askari, O. Weckner, H. Razi, S. A. Silling, Damage and failure analysis of composite laminates under biaxial loads, in: 47th AIAA Struct., Struct. Dyn., and Mater. Conf., Honolulu, HI, 2007.

[28] J. Xu, E. Askari, O. Weckner, S. A. Silling, Peridynamic analysis of impact damage in composite laminates, J. Aerosp. Engrg. 21 (2008) 187-194.

[29] B. Kilic, A. Agwai, E. Madenci, Peridynamic theory for progressive damage prediction in center-cracked composite laminates, Compos. Struct. 90 (2009) 141-151.

[30] E. Askari, J. Xu, S. A. Silling, Peridynamic analysis of damage and failure in composites, in: 44th AIAA Aerospace Sciences Meeting and Exhibition; No. 2006-88, Reno, Nevada, 2006.

[31] W. Hu, Y. D. Ha, F. Bobaru, Modeling dynamic fracture and damage in fiber-reinforced composites with peridynamics, Int. J. Multiscale Comput. Engrg. 9 (2011) 707-726.

[32] F. Bobaru, Y. D. Ha, Adaptive refinement and multiscale modeling in 2D peridynamics, Int. J. Multiscale Comput. Engrg. 9 (2011) 635-659.

[33] S. A. Silling, M. Zimmermann, R. Abeyaratne, Deformation of a peridynamic bar, J. Elasticity 27 (2003) 173-190.

[34] F. Bobaru, M. Yang, L. F. Alves, S. A. Silling, E. Askari, J. Xu, Convergence, adaptive refinement, and scaling in $1 \mathrm{D}$ peridynamics, Int. J. Numer. Meth. Engrg. 77 (2009) 852-877.

[35] S. A. Silling, E. A. Askari, Meshfree method based on the peridynamic model of solid mechanics, Comput. Struct. 83 (2005) 15261535.

[36] S. A. Silling, M. Epton, O. Weckner, J. Xu, E. Askari, Peridynamic states and constitutive modeling, J. Elasticity 88 (2007) 151-184.

[37] S. A. Silling, R. B. Lehoucq, Convergence of peridynamics to classical elasticity theory, J. Elasticity 93 (2008) 13-37.

[38] S. W. Yurgartis, Measurement of small angle fiber misalignments in continuous fiber composites, Compos. Sci. Technol. 30 (1987) 279-293.

[39] K. Ravi-Chandar, W. G. Knauss, An experimental investigation into dynamic fracture: IV. On the interaction of stress waves with propagating cracks, Int. J. Fract. 26 (1984) 189-200.

[40] S. Jose, R. R. Kumar, M. K. Jana, G. V. Rao, Intralaminar fracture toughness of a cross-ply laminate and its constituent sub-laminates, Compos. Sci. Technol. 61 (2001) 1115-1122.

[41] E. Hairer, C. Lubich, G. Wanner, Geometric numerical integration illustrated by the Strömer/Verlet method, Acta Numer. 12 (2003) $399-450$.

[42] W. Xie, Peridynamic flux-corrected transport algorithm for shock wave studies, Master Thesis, Department of Engineering Mechanics, University of Nebraska-Lincoln, Lincoln, Nebraska 2005. 